\title{
L'essai de cisaillement à la grande boîte de Casagrande : un banc expérimental pour évaluer les propriétés des sols grossiers et pour d'autres applications en géomécanique
}

\author{
Alain Bernard, Laurent Peyras ${ }^{1 \star}$ et Paul Royet \\ IRSTEA, 3275 route de Cézanne, CS 4061, 13182 Aix-en-Provence Cedex 5, France
}

\begin{abstract}
Résumé - L'article présente l'essai de cisaillement à la grande boîte de Casagrande à travers le banc expérimental développé par IRSTEA. Ce banc a été développé à l'origine pour l'étude des propriétés de résistance au cisaillement des sols grossiers. Il a connu au fil de ses perfectionnements des évolutions métrologiques et de pilotage qui permettent d'obtenir des lois de comportement et d'interfaces sous chemins de contraintes ou déformations contrôlés par ordinateur, la réalisation d'essais en conditions saturées, la détermination des contraintes résiduelles et la mise en œuvre d'essais cycliques. Des équipements particuliers ont été conçus pour répondre aux besoins spécifiques des essais d'interfaces géosynthétiques - géomatériaux et des dispositifs d'étanchéité par géosynthétiques. Par la suite, ce banc d'essai a été utilisé dans le cadre de projets de recherche et a permis des applications originales en géomécanique. L'article présente des travaux destinés à la caractérisation des propriétés de frottement de ballasts, à l'évaluation des conditions de stabilité sur pente des géosynthétiques, à la caractérisation de la résistance au cisaillement de bétons compactés au rouleau (BCR) et au renforcement des sols par la végétation.
\end{abstract}

Mots clés : grande boîte de cisaillement / boîte de cisaillement / cisaillement / matériaux grossiers / géosynthétique

\begin{abstract}
Casagrande large shear box test: an experimental test equipment to evaluate the properties of coarse soils and for other applications in geomechanics. The article presents the Casagrande large shear box test provided with the experimental test equipment developed by IRSTEA. This experimental test equipment was originally developed to study the shear strength properties of coarse soils in saturated conditions. It was then modified by metrological and monitoring enhancements. These improvements were necessary to evaluate the constitutive behaviour and interface laws under different stress paths, and cyclic loadings. Other special equipments were designed to meet the specific needs of interface testing between geomaterials and geosynthetics. Thereafter, this experimental test equipment was in the framework of a research project and for new applications in geomechanics. The article presents several studies to characterize the ballasts friction properties, to evaluate the conditions of geosynthetic slope stability, to characterize the roller compacted concrete (RCC) shear strength and the soil reinforcement with vegetation.
\end{abstract}

Keywords: large shear box / shear box / shearing / coarse material / geosynthetic

\section{Introduction}

L'étude de la résistance au cisaillement d'un sol constitue un problème complexe de la mécanique des sols. La résistance au cisaillement dépend du type de sol rencontré, suivant qu'il est cohérent ou non, que le matériau est grossier ou fin.

Dans la résolution des problèmes de stabilité en mécanique des sols, il est nécessaire de connaître les paramètres de résistance au cisaillement du sol. Ainsi, l'angle de frottement interne et la cohésion interviennent dans les calculs de stabilité des barrages ou des digues par la tangente de l'angle de frottement (notée tan $\Phi$ ) et par la cohésion effective (notée C) ou non drainée (notée $\mathrm{Cu}$ ), dans les calculs de poussée par le

^Auteur correspondant : laurent.peyras@irstea.fr coefficient de poussée active de la théorie de Rankine $\mathrm{K}_{\mathrm{a}}$, ou encore dans la justification des fondations superficielles par la contrainte de rupture du sol $\mathrm{q}_{\mathrm{u}}$.

Évaluer expérimentalement précisément les propriétés de résistance au cisaillement des sols constitue un enjeu important en mécanique des sols (Perrot, 1968). En effet, de petites variations sur l'angle de frottement interne et/ou sur la cohésion peuvent induire des dimensionnements sensiblement différents des ouvrages géotechniques, et par exemple, avoir des impacts importants sur les volumes de sols à mettre en œuvre pour la réalisation d'un barrage en terre ou d'une digue.

L'appareil expérimental le plus complet et privilégié en mécanique des sols pour évaluer les propriétés de résistance au cisaillement est l'essai triaxial. Il permet de contrôler la pression interstitielle (notée u) tout le long de l'essai et de mesurer la déformation axiale en fonction de la contrainte 
normale. Les cellules triaxiales courantes permettent de travailler sur des échantillons de $35 \mathrm{~mm}$ à $70 \mathrm{~mm}$ de diamètre.

Un essai alternatif à l'essai triaxial est l'essai de cisaillement rectiligne à la boîte. Il est plus facile à mettre en œuvre et est moins coûteux, mais présente les inconvénients vis-à-vis de l'essai triaxial, d'une part d'imposer un plan de cisaillement qui ne correspond pas à une direction principale, d'autre part de ne pas permettre de contrôler les pressions interstitielles durant l'essai. L'interprétation de l'essai est donc plus complexe et nécessite une analyse critique sur essais triaxiaux des valeurs obtenues (de calibration et/ou issus de la littérature). Les boîtes de cisaillement courantes permettent de travailler sur des échantillons de sections de $5 \times 5 \mathrm{~cm}^{2}$ à $10 \times 10 \mathrm{~cm}^{2}$.

Toutefois, compte tenu de la taille limitée des échantillons mis en œuvre dans ces deux types d'essais, ils restent dédiés à la recherche des propriétés de résistance au cisaillement des sols fins. En effet, le matériel d'essai doit prendre en compte la granulométrie de l'échantillon et en particulier la dimension du plus gros élément contenu dans le matériau doit tenir compte de la taille du boîtier d'essai. Par ailleurs, l'écrêtage des matériaux et l'extrapolation ensuite des résultats expérimentaux pour des matériaux plus grossiers conduisent à augmenter l'incertitude sur les résultats. La recommandation habituelle conduit à considérer que la taille du boîtier d'essai doit au moins être égale à environ 5 à 6 fois la taille du plus gros grain contenu dans l'échantillon de sol.

On constate que les sols grossiers ont une place prépondérante dans les ouvrages de génie civil. Historiquement, l'étude des sols grossiers a été directement liée à la réalisation des grands barrages en terre au siècle dernier, qui a vu le développement d'équipements créés spécifiquement pour répondre aux contraintes techniques posées par la réalisation d'ouvrages en sols grossiers (CIGB, 1993 ; Manba, 1989).

Les travaux engagés par la communauté géotechnique sur le sujet de la caractérisation de la résistance au cisaillement des sols grossiers sont motivés par un besoin d'apporter des connaissances nouvelles sur les principales questions suivantes (CIGB, 1993 ; Marsal et Fuentes de la Rosa, 1976) :

- quels sont les paramètres géomécaniques essentiels qui influencent la résistance au cisaillement des sols grossiers ?

- quel est le rôle de l'eau dans le comportement au cisaillement d'un sol grossier?

- quelle est l'influence du pourcentage d'éléments fins par rapport à la proportion de sols à forte granularité ? Comment agit la matrice cohésive d'un sol composé d'une fraction fine et d'éléments grossiers?

À côté des questions scientifiques, il est une question d'ordre technique qui est de savoir comment caractériser la résistance au cisaillement d'un sol grossier à partir d'appareillages d'essais de laboratoire adéquats.

Le premier équipement est le dispositif triaxial de révolution. Ces appareils sont des prototypes et il n'existe à notre connaissance que quelques équipements en France, notamment une cellule triaxiale de diamètre $300 \mathrm{~mm}$ au laboratoire Navier de l'université Paris Est (Pedro, 2004) et au bureau d'études géotechnique Fugro Paris, et un triaxial de diamètre $1 \mathrm{~m}$ à l'école centrale de Nantes. De façon analogue aux essais sur sols fins, les essais réalisés à partir de grands appareils triaxiaux donnent en principe les meilleurs résultats pour la caractérisation des propriétés de résistance au cisaillement du sol grossier, car ils permettent de contrôler les pressions interstitielles lors du cisaillement du sol. Toutefois, leur réalisation est très onéreuse, et les essais sont difficiles à mettre en œuvre vu leurs tailles et ne peuvent être rentables que pour des études de grands projets géotechniques tels que les grands barrages en terre, justifiant d'importants investissements matériels et humains.

Le deuxième type d'équipement de grande dimension est la grande boîte de cisaillement rectiligne, typiquement de dimension $0,50 \times 0,50 \times 0,50 \mathrm{~m}^{3}$ à $1,00 \times 1,00 \times 0,50 \mathrm{~m}^{3}$. Ces essais sont plus faciles à réaliser et à mettre en œuvre que ceux sur les grands appareils triaxiaux. Même si la grande boîte de cisaillement n'offre pas toutes les possibilités d'un équipement triaxial, elles peuvent permettre selon leur sophistication de réaliser des essais de cisaillement de sols grossiers en condition non saturée ou saturée, de réguler en vitesse le cisaillement et de piloter l'essai en chemins de contrainte complexes. La possibilité de réaliser des essais en conditions saturées sur des échantillons préalablement consolidés représente une possibilité intéressante.

Aujourd'hui, seulement quelques laboratoires en France sont équipés ou ont développé de tels dispositifs expérimentaux dédiés aux sols grossiers. On peut notamment citer :

- le laboratoire du CEREMA Lyon qui possède une boîte cylindrique de $60 \mathrm{~cm}$ de diamètre et de $50 \mathrm{~cm}$ de hauteur ; - l'INSA de Lyon dispose d'une boîte parallélépipédique de dimension $60 \times 60 \times 40 \mathrm{~cm}^{3}$ (Bourdeau, 1997);

- le CER de Rouen qui a construit plus récemment une boîte parallélépipédique de $50 \times 50 \times 50 \mathrm{~cm}^{3}$.

Le laboratoire de géomécanique d'IRSTEA - anciennement Cemagref - s'intéresse depuis son origine à la question de l'évaluation des ouvrages hydrauliques tels que les barrages (Serre et al., 2007). Il a développé au début des années 1980 une boîte de cisaillement rectiligne de grande dimension - appelé dans la suite de l'article « grande boîte de Casagrande »-, destinée à l'origine à caractériser les propriétés de résistance au cisaillement des sols grossiers dans un cadre de conception de barrages en aménagement rural.

Cet équipement a progressivement évolué au cours des années, en termes technologiques (pilotage, métrologie, etc.), mais aussi en termes d'applications et de vocation, passant progressivement d'un banc d'essai de bureau d'études à un banc d'essai expérimental utilisé dans le cadre de programmes de recherche.

Le présent article présente la grande boîte de Casagrande. Dans une première partie, il décrit la conception technologique du banc d'essai et de ses améliorations tout le long des 30 années d'usage pour la caractérisation des propriétés des sols grossiers, avec une application type sur des matériaux grossiers. Les parties suivantes traitent d'autres applications en géomécanique du dispositif expérimental conduites dans le cadre de projets de recherche :

- la caractérisation des propriétés de frottement de ballasts SNCF ;

- l'évaluation des conditions de stabilité sur pente des géosynthétiques ;

- la caractérisation de la résistance au cisaillement de bétons compactés au rouleau (BCR) ;

- le renforcement des sols par la végétation. 


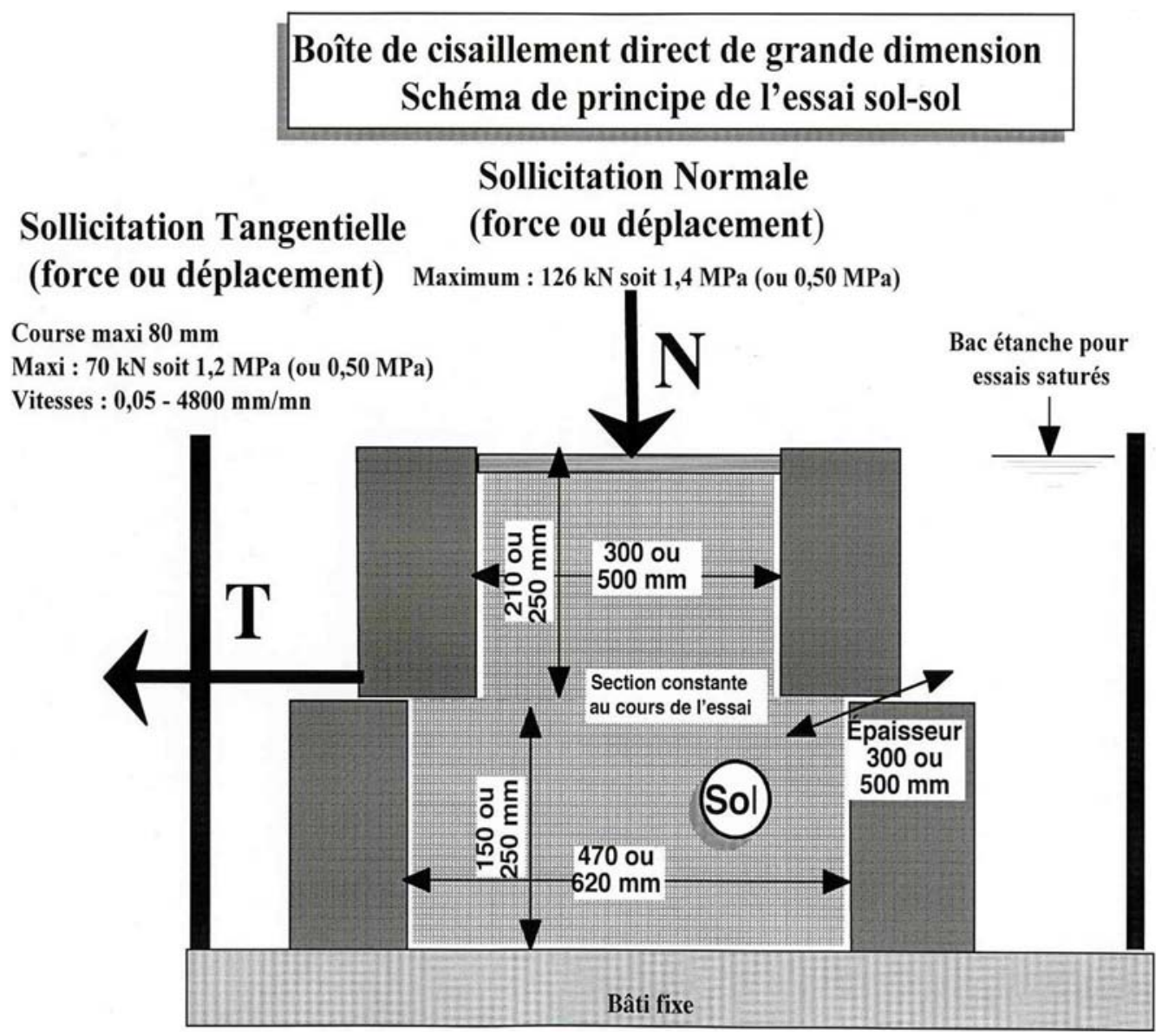

Fig. 1. Schéma de la grande boîte de Casagrande.

Fig. 1. Schematic view of the Casagrande large shear box.

L'objectif de l'article est de dresser un panorama des différentes applications de recherche mises en œuvre avec la grande boîte de Casagrande à IRSTEA. Il ne s'agit pas, pour chacune des applications, de montrer et discuter les détails des projets de recherche conduits, mais plutôt d'exposer le contexte de ces applications, les principes expérimentaux mis en œuvre au moyen de la grande de boîte de cisaillement et les principaux résultats obtenus. L'article vise donc à montrer comment la grande boîte de Casagrande a pu être exploitée et adaptée dans différentes applications de la géomécanique.

Les perspectives de recherche et développement autour de la caractérisation de la résistance au cisaillement des sols grossiers sont discutées en conclusion.

\section{Développement d'une grande boîte de Casagrande}

Afin de répondre à la demande d'essais représentatifs des sols grossiers, le laboratoire de géomécanique d'IRSTEA a développé une boîte de cisaillement rectiligne de grandes dimensions permettant de travailler principalement avec des matériaux remaniés contenant des granulats de grande taille de l'ordre de 60 à $80 \mathrm{~mm}$ et plus suivant la proportion, mais aussi d'étudier les lois d'interfaces entre différents géomatériaux. Deux boîtiers de cisaillement rectiligne de type Casagrande de dimension $30 \times 30 \times 30 \mathrm{~cm}^{3}$ et $50 \times 50 \times 50 \mathrm{~cm}^{3}$ ont ainsi été développés.

Cette partie se propose de détailler le dispositif expérimental de cisaillement. Elle traite ensuite du protocole expérimental relatif à la mise en place des matériaux dans les boîtiers de cisaillement. Elle présente les questions de l'instrumentation installée sur l'équipement de la grande boîte qui permet le pilotage informatique et l'asservissement électrohydraulique qui en découle. Dans le cadre d'une démarche qualité, le suivi métrologique des capteurs et le calcul des incertitudes sur les résultats des essais font l'objet d'une attention particulière ; de même, la mise en place de nouvelles réglementations en hygiène et sécurité a permis d'optimiser le fonctionnement de la machine ainsi que la qualité d'exécution des essais par les opérateurs.

\subsection{Description du dispositif d'essai}

La grande boîte de Casagrande est constituée de deux demiboîtes dont l'une, la boîte supérieure, peut glisser sur l'inférieure le long d'un plan de cisaillement horizontal suivant une course de $80 \mathrm{~mm}$ (Fig. 1). 
Le boîtier inférieur a des dimensions supérieures aux dimensions du boîtier supérieur, ceci afin de conserver la même section de cisaillement durant le déroulement de l'essai.

L'appareillage se compose en outre :

- d'une centrale hydraulique qui permet la stabilisation et la régulation des efforts de confinement et de cisaillement à partir des servo valves de régulation ;

- d'un bâti métallique rigide support des vérins hydrauliques asservis, qui transmettent les efforts de compression verticale et de cisaillement horizontal ;

- d'un ensemble micro-ordinateur et automate qui pilote les essais de cisaillement.

\section{L'équipement offre de nombreuses possibilités :}

- il permet d'obtenir des lois de comportement et d'interfaces sous chemins de contraintes ou déformations contrôlés par ordinateur. L'essai est utilisé classiquement selon la norme NF P 94-071-1 : vitesse de déplacement constante et charge normale constante. Toutefois, ce banc permet également des chemins de contraintes plus complexes tels que les chargements cycliques ou monotones (croissant avec le temps) et des déplacements cycliques. Néanmoins, l'asservissement ne permet pas de travailler à condition de raideur constante. Les exemples développés dans l'article concernent l'utilisation dans les conditions classiques de vitesse de déplacement constant et de charge normale constante ;

- la mise en place d'un caisson permet la réalisation d'essais en conditions saturées ;

- le système de supervision permet la détermination des contraintes résiduelles et la mise en œuvre d'essais cycliques ;

- des équipements particuliers ont été conçus pour répondre aux besoins spécifiques des essais d'interfaces géosynthétiques/géomatériaux et des dispositifs d'étanchéité par géosynthétiques (DEG).

\subsection{Mise en place des sols}

Le sol mis en place dans la boîte de cisaillement a une densité correspondant à celle de l'Optimum Proctor Normal ou à celle requise spécifiquement pour les besoins de l'essai. Pour atteindre la densité requise, on compacte le sol en plusieurs couches (trois ou cinq), soit par pressage statique, soit par compactage vibratoire (compacteur vibrant). L'objectif est de mettre le plan de cisaillement dans le milieu d'une couche. En outre, chaque couche est constituée de façon à reproduire en son sein les conditions de densité recherchées (essai Proctor ou contraintes visées sur le chantier). Ainsi verticalement, l'échantillon est considéré comme homogène au sein de ses 3 ou 5 couches.

Le pressage statique est appliqué par le vérin utilisé pour transmettre l'effort normal lors de l'essai de cisaillement. On augmente la force appliquée jusqu'à ce que la couche à compacter ait l'épaisseur correspondante à la densité requise. On procède de même pour le vibratoire.

Pour déterminer les caractéristiques internes d'un sol, on procède de même dans les deux demi-boîtes superposées, mais avec un nombre total de couches impair de telle sorte que le plan de reprise d'une des couches ne se trouve pas dans le plan de cisaillement. Un soin particulier est pris pour la réalisation des débords de la boîte inférieure.

\subsection{L'instrumentation, le pilotage et l'asservissement de la grande boîte de Casagrande}

La grande boîte de Casagrande est équipée en capteurs de force et de déplacement.

Les capteurs de force permettent :

- la mesure de la force de cisaillement au moyen d'un capteur horizontal de $200 \mathrm{kN}$;

- la mesure de la force nécessaire à l'obtention de la pression de confinement au moyen de deux capteurs de force verticaux de $100 \mathrm{kN}$ agissant en sommation.

Un couple de capteurs de déplacement de haute précision est chargé de la mesure des déplacements suivant les deux axes :

- le capteur horizontal, de type règle numérique Magnescale, enregistre les déplacements du boîtier supérieur et permet la régulation en vitesse de ce boîtier ;

- le capteur vertical, également de type règle numérique, mesure la contractance ou la dilatance générale des matériaux pendant l'essai.

Le dispositif est équipé de deux vérins hydrauliques de $12000 \mathrm{dN}$ de capacité maximale, générant les mouvements de déplacement du boîtier et de compression du matériau. Une centrale hydraulique fournit une pression de 160 bars au réseau de servo valves et aux vérins.

L'automate électronique programmable associé aux conditionneurs affectés aux différents capteurs réalise l'interface entre le micro-ordinateur de pilotage et les servo valves qui génèrent les mouvements des vérins en force et en déplacement.

L'ensemble constitué des boîtiers, vérins, servo valves, capteurs est géré par un système de supervision permettant le pilotage à partir d'un logiciel développé par IRSTEA et l'acquisition des données d'essais qui se fait à partir du poste micro-ordinateur en provenance de l'automate programmable (Omronau standard OPC de type Sysmac C200HE). Le protocole de communication entre le logiciel et l'automate est de type Modbus.

Les principes de l'instrumentation, pilotage et asservissement de la grande boîte de Casagrande sont résumés sur la Figure 2.

Les asservissements de l'axe horizontal (cisaillement) et de l'axe vertical (compression/confinement) sont gérés de façon à être indépendants. Ainsi, chaque axe peut être asservi en force ou en position.

L'essai de cisaillement rectiligne classique correspond à un asservissement réalisé avec une contrainte de confinement constante pendant tout le déroulement de l'essai et l'axe horizontal est asservi en vitesse de déplacement constante.

La précision de l'asservissement autorise un réglage de la contrainte sur l'échantillon pouvant varier de 0 à $1200 \mathrm{kPa}$ avec une précision de plus ou moins $0,30 \mathrm{kPa}$ sur toute l'échelle. En ce qui concerne les déplacements, les règles Magnescales délivrent des signaux de type incrémentaux (précision de base : $1 / 100 \mathrm{~mm}$ ) et elles sont réglées pour donner une précision des mesures de l'ordre du $1 / 10^{\text {ème }}$ de millimètre. 


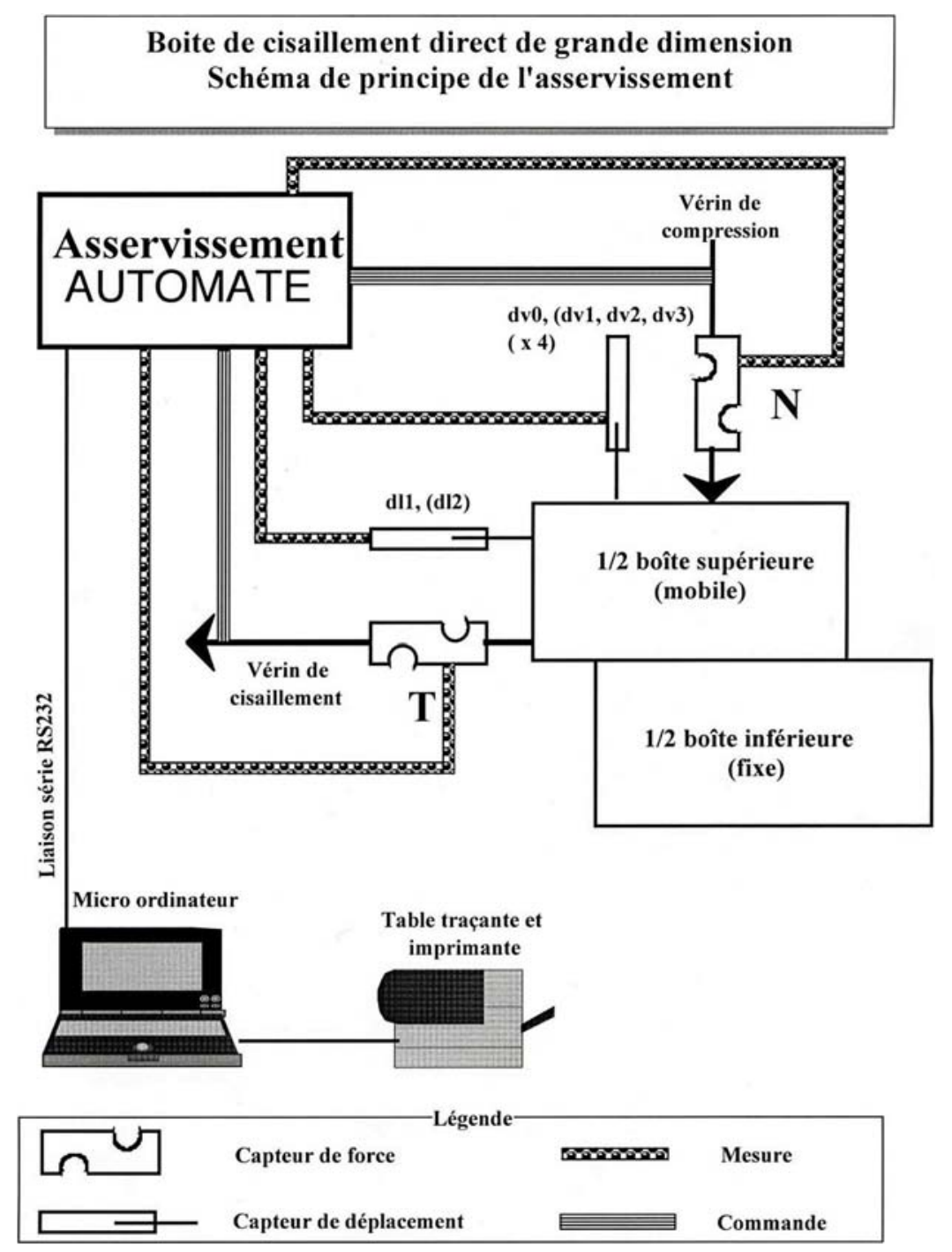

Fig. 2. Asservissement et pilotage de la grande boîte de Casagrande.

Fig. 2. Control and monitoring of the Casagrande large shear box.

\subsection{Métrologie et calculs des incertitudes}

En octobre 2005, IRSTEA a fait procéder à un audit diagnostic technique relatif à la métrologie de la grande boîte de Casagrande, dans l'objectif d'établir une analyse critique de la métrologie de la boîte et une évaluation des incertitudes pesant sur les résultats des essais.

La démarche d'évaluation des incertitudes a compris :

- une analyse du processus de mesure (moyens, méthode, matière, milieu et main d'œuvre) ;

- une évaluation des incertitudes types ;

- l'établissement de la loi de propagation des incertitudes ;

- l'adoption de l'incertitude élargie.

Les conclusions de cet audit ont été l'estimation de l'incertitude des résultats :

- l'angle de frottement $\Phi$ est obtenu avec une précision de plus ou moins $1,2^{\circ}$;
- la cohésion $\mathrm{C}$ est obtenue avec une précision de plus ou moins $8,4 \mathrm{kPa}$.

Ces données d'incertitude sont prises en compte dans les essais normalisés et de recherche conduits à la grande boîte de Casagrande.

\section{5 Évolution de la grande boîte de Casagrande en matière d'hygiène et de sécurité}

Suite aux recommandations faites par le document unique d'évaluation des risques professionnels, le laboratoire de géomécanique d'IRSTEA a pris la décision de faire réaliser les travaux nécessaires à la suppression du risque résiduel de chute (qualifié de très fort) présent lors des manipulations sur cet équipement. Pour cela, l'opération a consisté à réaliser une fosse dans le vide sanitaire du laboratoire et y placer la grande boîte. Ainsi, le plan de travail de l'opérateur a été descendu de telle sorte qu'il se situe à une hauteur allant de 70 à $110 \mathrm{~cm}$, 


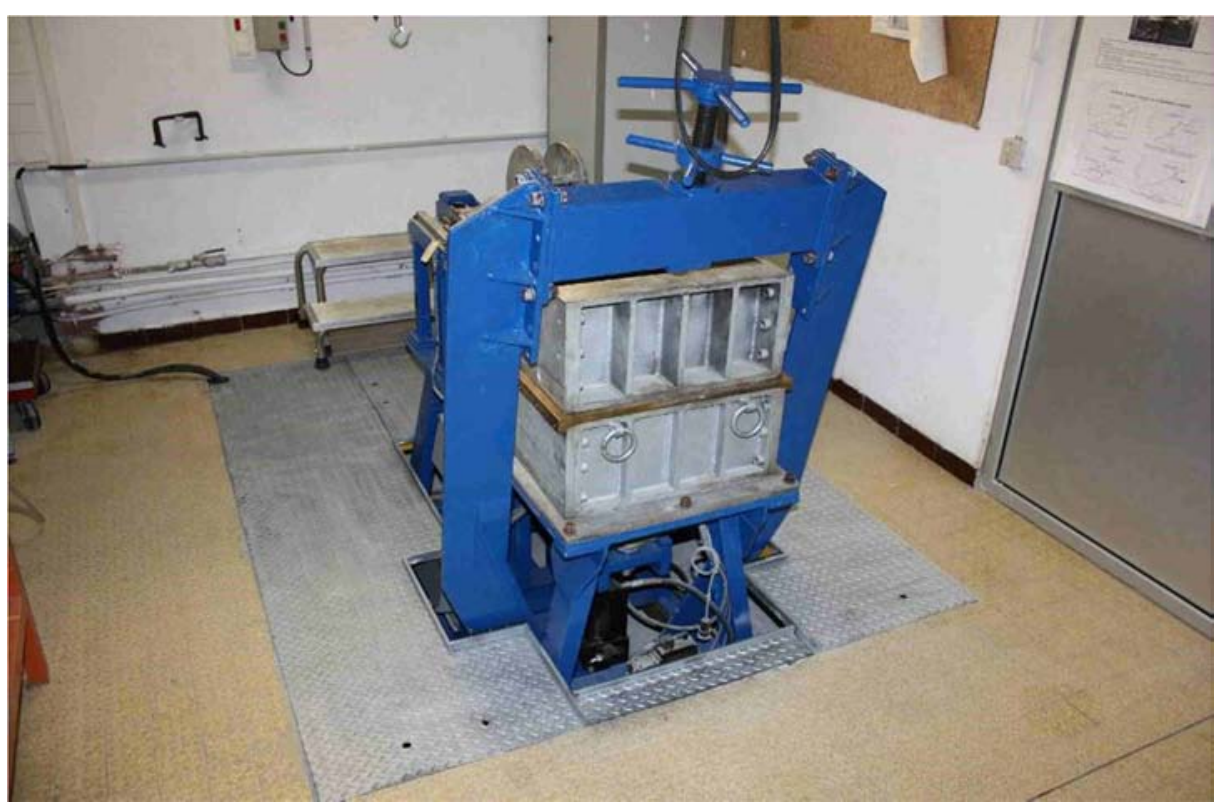

Fig. 3. Banc d'essai de la grande boîte de Casagrande dans sa configuration actuelle.

Fig. 3. The Casagrande large shear box in its current configuration.

correspondant à la majorité des opérations réalisées sur cet appareillage répondant à la norme NF 141222-2 (Fig. 3).

\subsection{Un exemple type d'essai réalisé à la grande boîte de Casagrande sur des sols grossiers}

La vocation initiale de la grande boîte de Casagrande est de réaliser des essais sur les sols grossiers. Ces matériaux sont souvent présents dans les sites montagnards et nous avons réalisé plusieurs campagnes d'essais sur des sols grossiers en provenance de ces sites (Gotteland et al., 2000 ; Tacnet et al., 2000). Nous présentons ci-après une illustration d'essais conduits sur un sol grossier lors d'études de faisabilité d'ouvrages de génie civil. Cet exemple est issu d'un projet de barrage d'altitude dans les Hautes Alpes (France).

L'objectif de l'étude a consisté à identifier et caractériser le matériau, une grave limono argileuse $0 / 80 \mathrm{~mm}$, prélevé à proximité de la zone de réalisation de l'ouvrage. Le programme d'essais convenu prévoyait la réalisation d'essais d'identification classiques des matériaux - teneur en eau, analyse granulométrique (tamisage et sédimentation), limite d'Atterberg et bleu de méthylène - et des essais de cisaillement à la grande boîte de Casagrande.

La détermination granulométrique a fait apparaître des granulats ayant une taille maximum avoisinant les $50 \mathrm{~mm}$ (Fig. 4). La grande boîte de Casagrande équipée du boîtier $50 \times 50 \mathrm{~cm}^{2}$ est apparue adaptée pour déterminer les caractéristiques de résistance au cisaillement $\mathrm{C}$ et $\tan \Phi$ d'un tel matériau.

À partir des informations relatives à la granulométrie et à la densité, les essais de cisaillement sont entrepris en mettant le matériau en place dans le boîtier $50 \times 50 \mathrm{~cm}^{2}$ à l'aide du compacteur vibrant (Fig. 5). Le matériau est mis en place en trois couches, compactées, puis scarifiées aux inter-couches, de telle sorte à obtenir la densité désirée en vérifiant que la masse de sol rentre bien dans le volume défini par le calcul. La couche située au niveau du plan de cisaillement est intégrée aux deux demi-boîtiers en une seule phase de compactage.

La pression de confinement est appliquée au sol par le vérin vertical en utilisant comme intermédiaire une plaque d'application des contraintes de section carrée, adaptée à la taille du boîtier supérieur $\left(30 \times 30 \mathrm{~cm}^{2}\right.$ ou $\left.50 \times 50 \mathrm{~cm}^{2}\right)$. Une fois le vérin mis en contact, l'essai est entièrement piloté par informatique et suit la procédure choisie (vitesse de cisaillement, force de confinement, etc.).

Classiquement, quatre essais de cisaillement correspondant à des pressions de confinement différentes $(100 \mathrm{kPa}, 200 \mathrm{kPa}$, $300 \mathrm{kPa}$ et $400 \mathrm{kPa}$ ) sont réalisés pour le même matériau. Ils permettent de déterminer les courbes contraintes-déformation et tassement-gonflement des matériaux (Fig. 6).

Les quatre courbes obtenues montrent que, dans chacun des cas, la résistance au cisaillement maximale est atteinte entre 30 et $40 \mathrm{~mm}$ de déplacement, correspondant à $6 \%$ et $8 \%$ de déplacement relativement à la taille de l'échantillon. Ce maximum passé, il est constaté un palier de la résistance au cisaillement et un comportement correspondant à la résistance résiduelle du sol.

Les courbes tassement/gonflement sont représentatives des mouvements inter-granulaires des graves présentes dans le sol. Dans cet exemple, nous constatons une phase de contractance allant jusqu'à $5 \%$ de déplacement, puis un processus de dilatance s'engage jusqu'à l'arrêt de l'essai. Les oscillations visibles sur la Figure 7 sont expliquées par des réagencements des grains durant l'essai de cisaillement, ces réarrangements de grains se produisant rapidement au regard de la vitesse de déplacement imposée dans l'essai.

Les courbes contraintes-déformations sont ensuite interprétées dans le diagramme de Mohr pour permettre de déterminer la cohésion et l'angle de frottement du matériau (Fig. 7). 


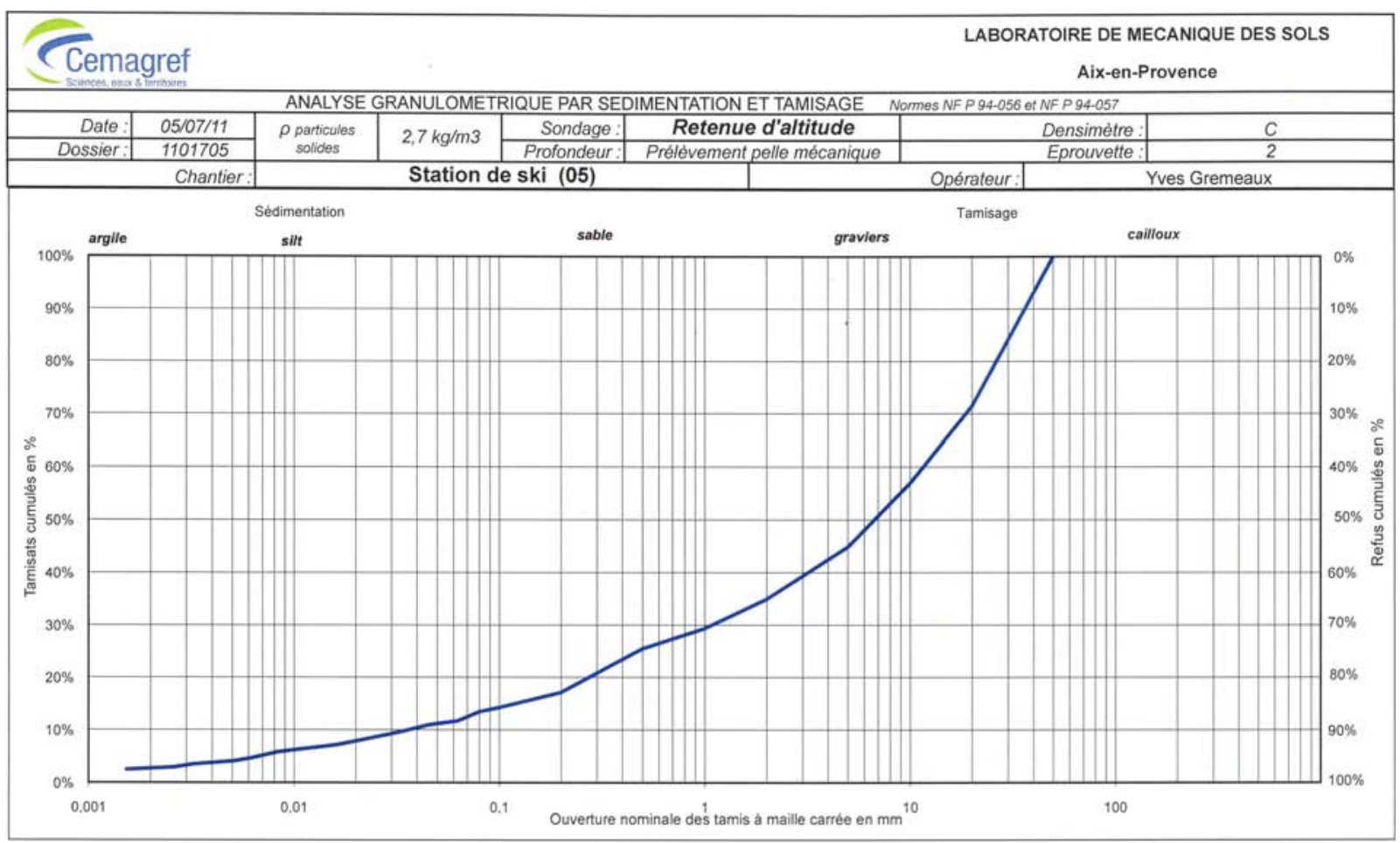

Fig. 4. Courbe granulométrique du matériau en provenance du site du barrage.

Fig. 4. Grain size distribution of the dam site material.

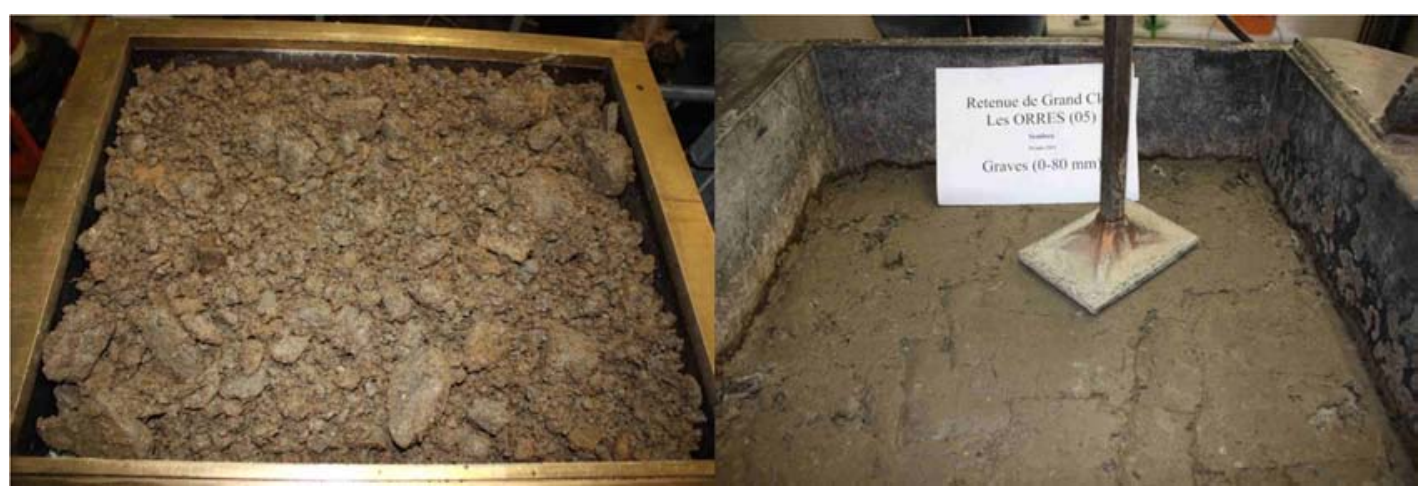

Fig. 5. Matériau foisonné dans le demi-boîtier inférieur (gauche) et matériau compacté dans le demi-boîtier supérieur (droite).

Fig. 5. Non-compacted material in the lower box (left) and compacted material in the upper box (right).

Dans cet exemple, les quatre points d'essais sont correctement alignés sur une même droite et ne nécessitent pas d'essais supplémentaires. Les valeurs de $\mathrm{C}$ et de $\tan \Phi$ présentées sur la Figure 7 correspondent aux valeurs de résistance au cisaillement maximales. L'essai est également interprété pour des déplacements de $1 \%(5 \mathrm{~mm}), 5 \%(25 \mathrm{~mm})$ et $10 \%(50 \mathrm{~mm})$.

\section{3 Étude de la caractérisation de ballasts SNCF}

\subsection{Contexte}

En 2003, le laboratoire de géomécanique d'IRSTEA a conduit une étude de recherche et développement avec la SNCF visant à caractériser le comportement des ballasts des lignes
LGV. L'objectif de l'étude était de vérifier l'influence de la compacité et de la saturation sur les caractéristiques de résistance au cisaillement $\mathrm{C}$ et $\tan \Phi$ de ces matériaux.

Pour conduire ce programme, IRSTEA a engagé une série d'essais sur des ballasts granitiques utilisés sur les lignes LGV. Les essais ont été effectués sur des matériaux extraits de deux carrières fournissant les matériaux à la SNCF. Il s'agissait des carrières de la Gouraudière et de Cusset, situées géologiquement dans des zones où l'on trouve les granites parmi les plus performants en France, présentant un poids volumique moyen du grain de $26,7 \mathrm{kN} / \mathrm{m}^{3}$ pour les deux carrières.

Les mesures des poids volumiques initiaux foisonnés mesurées sur les ballasts sont les suivants :

- ballast de la Gouraudière : $13,47 \mathrm{kN} / \mathrm{m}^{3}$;

- ballast de Cusset : $13,27 \mathrm{kN} / \mathrm{m}^{3}$. 
Cemagref d'Aix en Provence
Essai de cisaillement à la grosse boîte de $50 \times 50 \mathrm{~cm} 2$ Échantillon de grave limono-sableuse Retenue d'altitude (05)

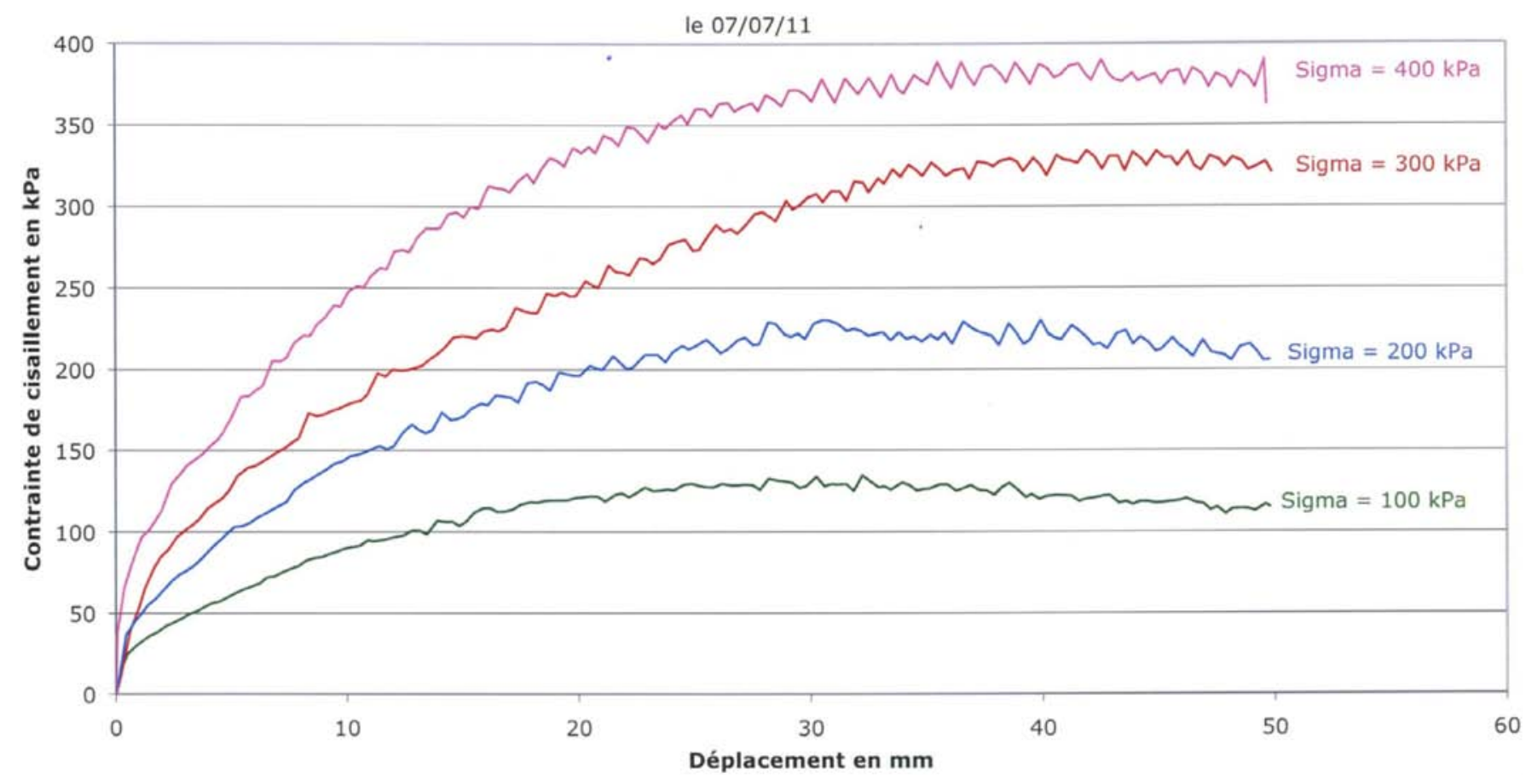

Cemagref d'Aix en Provence Essai de cisaillement à la boîte de $50 \times 50 \mathrm{~cm} 2$ Échantillon de grave limono-sableuse Retenue d'altitude (05)

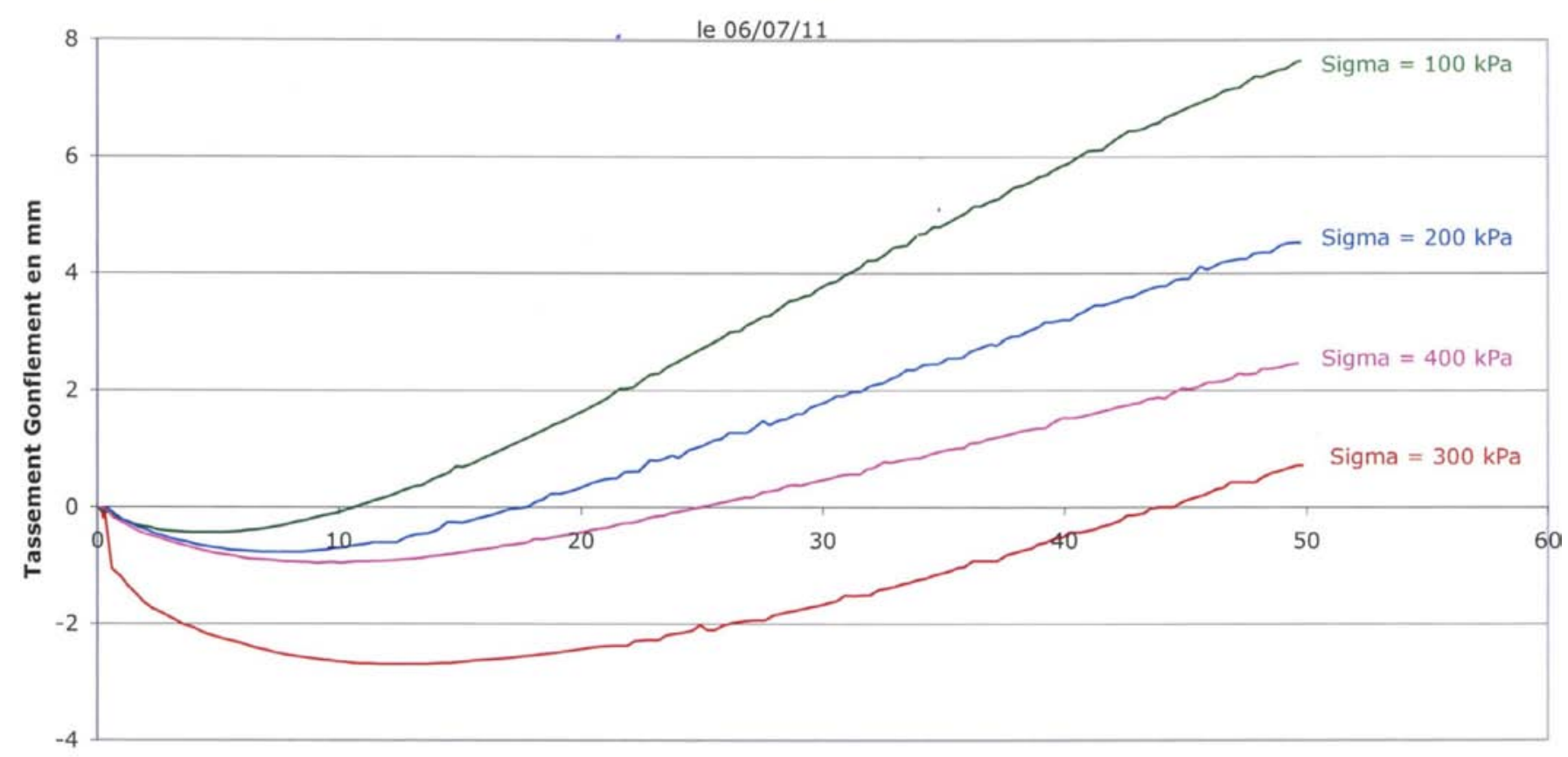

Déplacement en $\mathrm{mm}$

Fig. 6. Courbes contraintes/déformation (gauche) et tassement/gonflement (droite) de la grave limoneuse.

Fig. 6. Stress/strain curves (left) and settlement/swelling (right) of the silts. 


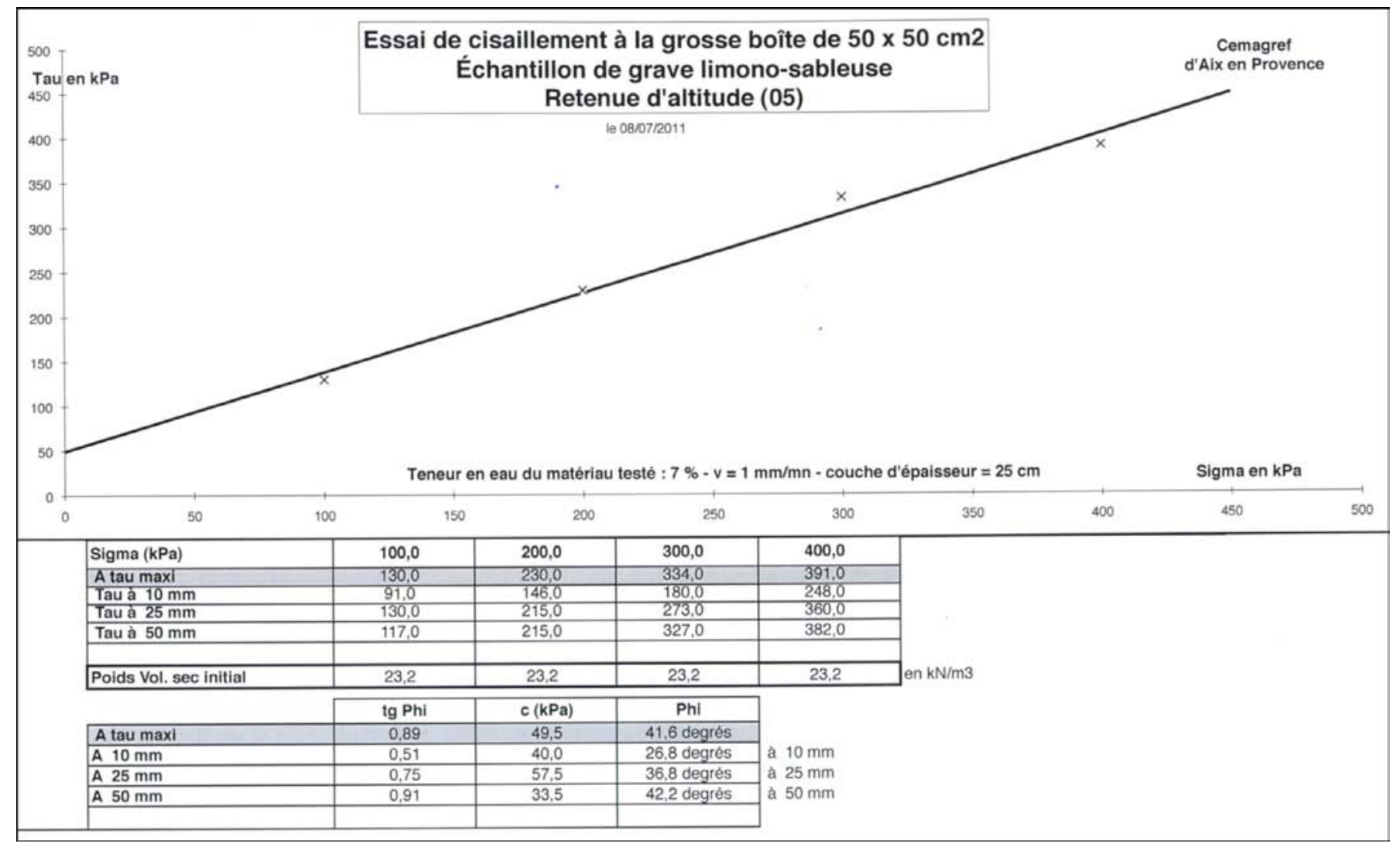

Fig. 7. Interprétation suivant la droite de Coulomb.

Fig. 7. Interpretation following Coulomb's model.

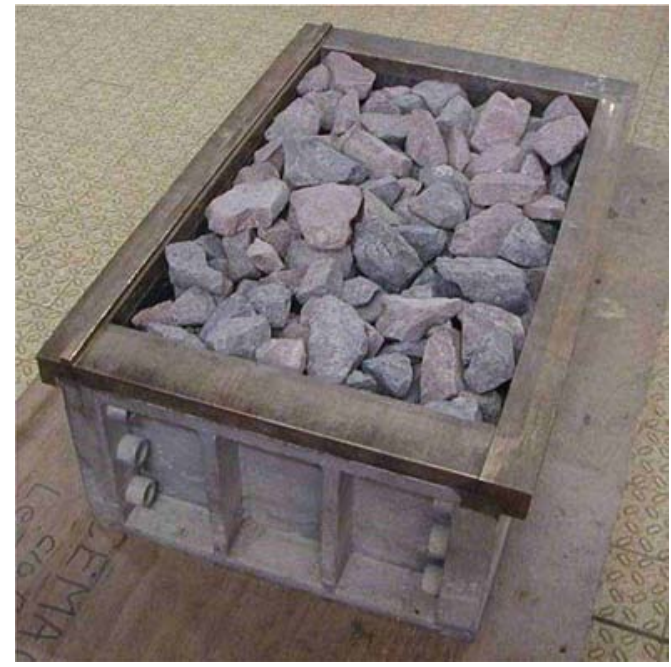

Fig. 8. Mise en place des ballasts en condition foisonnée.

Fig. 8. Placing ballasts in non-compacted conditions.

\subsection{Expérimentations conduites à la grande boîte de Casagrande}

Trois modes de préparation des ballasts correspondant à trois compacités ont été employés :

- le premier mode consistait à verser directement les matériaux dans les boîtiers de cisaillement sans compactage : ces essais sont dits en « condition foisonnée » (Fig. 8) ;

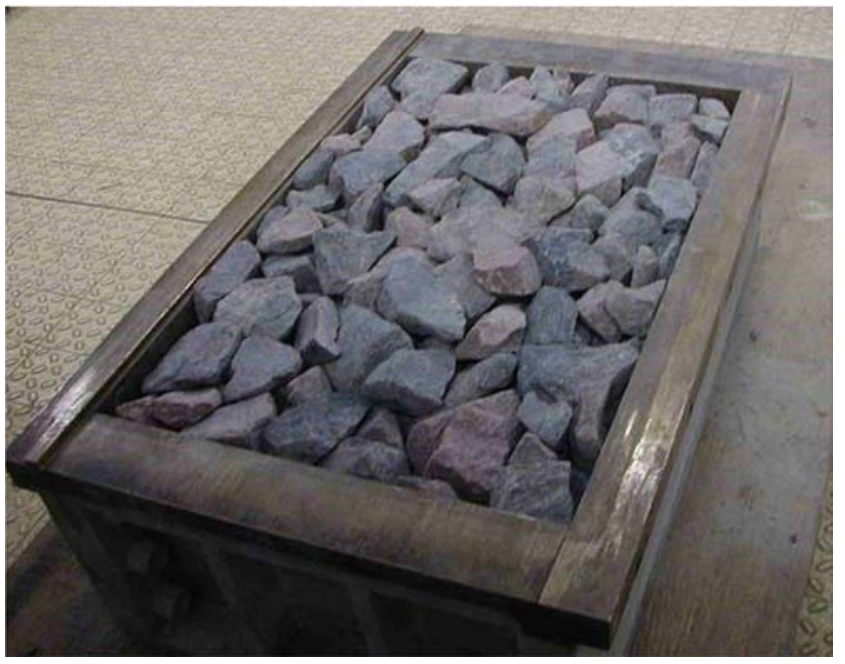

Fig. 9. Mise en place des ballasts en condition agencée.

Fig. 9. Placing ballasts in orderly conditions.

Les mesures des poids volumiques en « condition foisonnée » effectuées sur les matériaux ont donné les résultats suivants :

- ballast de la Gouraudière : $13,27 \mathrm{kN} / \mathrm{m}^{3}$,

- ballast de Cusset : $13,11 \mathrm{kN} / \mathrm{m}^{3}$;

- le deuxième mode dit en « condition agencée » (Fig. 9) : compte tenu de la difficulté de mettre en œuvre au laboratoire 


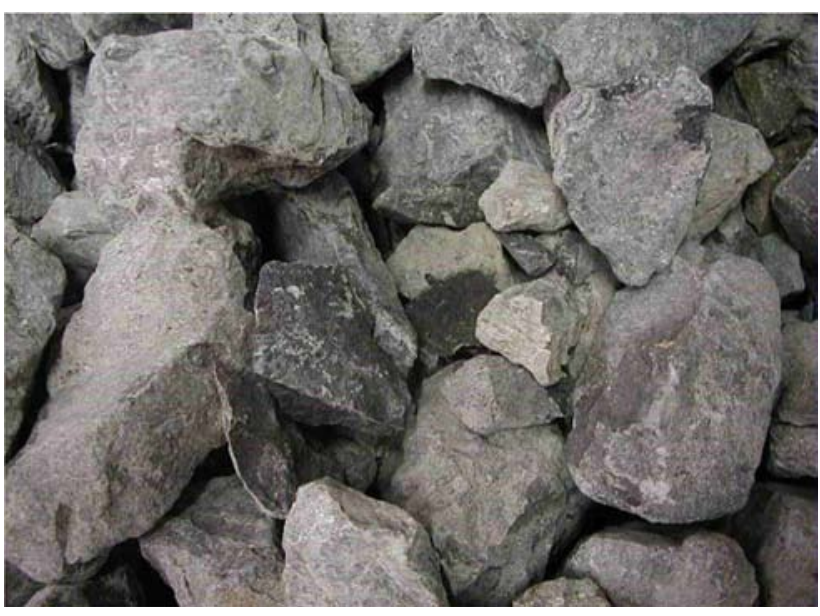

Fig. 10. Brisures des ballasts compactés au compacteur vibratoire.

Fig. 10. Broken compacted ballasts with vibratory compactor.

un compactage équivalent à celui utilisé par la SNCF pour compacter leurs ballasts, nous rangeons les éléments du ballast de telle sorte à ce que chacun d'entre eux s'imbrique parfaitement l'un par rapport à l'autre, de manière à diminuer l'indice des vides au maximum.

Les mesures des poids volumiques en " condition agencée » effectuées sur les matériaux ont donné les résultats suivants :

- ballast de la Gouraudière : $16,91 \mathrm{kN} / \mathrm{m}^{3}$,

- ballast de Cusset : $16,38 \mathrm{kN} / \mathrm{m}^{3}$;

- le troisième mode dit en " condition compactée » a pour objectif de compacter les graves avec un compacteur vibratoire à aiguille. Cette procédure ayant pour objectif de se rapprocher le plus possible de la méthode utilisée par la SNCF sur ses ballasts de voies ferrées, elle est de nature à briser des éléments de ballasts et donc à modifier potentiellement la granulométrie du matériau (Fig. 10).

Les mesures des poids volumiques en « condition compactée » effectuées sur les matériaux ont donné les résultats suivants :

- ballast de la Gouraudière : $15,56 \mathrm{kN} / \mathrm{m}^{3}$,

- ballast de Cusset : $15,39 \mathrm{kN} / \mathrm{m}^{3}$.

\subsection{Résultats des essais de cisaillement sur les graves $25 / 50 \mathrm{~mm}$}

Pour vérifier l'importance de l'effet de l'imbibition superficielle sur le frottement inter-granulaire, nous avons procédé à des essais de cisaillement sous conditions sèche et saturée. Ces essais ont été réalisés dans la boîte de Casagrande de $30 \times 30 \mathrm{~cm}^{2}$ et à la vitesse de cisaillement de $1 \mathrm{~mm} / \mathrm{min}$. Ils ont permis d'obtenir les courbes contraintes-déformations des matériaux dans les différents états de conditionnement. Lors de l'essai de cisaillement, le matériau montre une évolution de l'augmentation en dent de scie de la contrainte de cisaillement avec le déplacement, caractérisant le réarrangement permanent des éléments de ballast dans la boîte de cisaillement et conduisant alors à la baisse brutale ponctuelle de la résistance au cisaillement. Lors des différents essais, nous obtenons, pour les quatre contraintes de confinement adoptées $(50 \mathrm{kPa}$, $100 \mathrm{kPa}, 200 \mathrm{kPa}$ et $400 \mathrm{kPa}$ ), un palier permettant de déterminer une valeur de résistance au cisaillement résiduelle (Fig. 11).

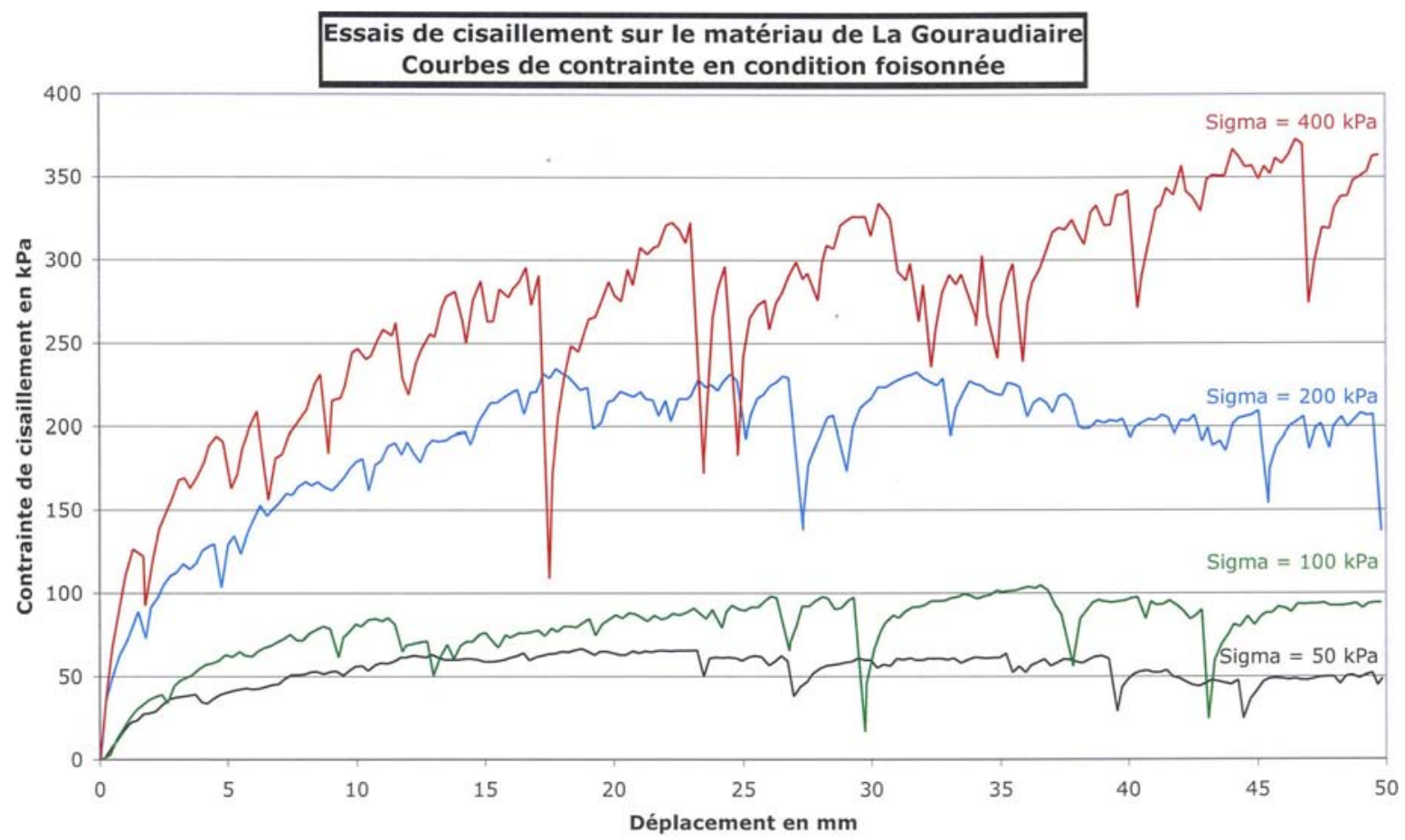

Fig. 11. Courbes contraintes/déformations du matériau de la Gouraudière en condition foisonné.

Fig. 11. Stress/strain curves of the Gouraudière material in non-compacted conditions. 
Tableau 1. Résultats des essais de cisaillement en fonction des conditions de mise en œuvre.

Table 1. Results of the shear tests vs. the implementation conditions.

\section{Essais de cisaillement sur ballast granitique LGV de 25/50 mm}

\begin{tabular}{|c|c|c|c|c|c|}
\hline & \multicolumn{5}{|c|}{ 1) Condition foisonné } \\
\hline & Site d'origine & & $\Phi$ en $^{\circ}$ & C en $\mathrm{kPa}$ & Tg Phy \\
\hline \multirow{5}{*}{ SEC } & \multirow[t]{3}{*}{ Cusset } & maximum & 41,5 & 58,6 & 0,88 \\
\hline & & palier & 39,9 & 35,7 & 0,84 \\
\hline & & & & & \\
\hline & \multirow[t]{2}{*}{\begin{tabular}{|l|} 
La Gouraudiaire \\
\end{tabular}} & maximum & 41,4 & 27,2 & 0,88 \\
\hline & & palier & 40,8 & 10,4 & 0,86 \\
\hline \multirow{5}{*}{ SATURÉ } & \multirow[t]{3}{*}{ Cusset } & maximum & 38,5 & 37,4 & 0,79 \\
\hline & & palier & 37,4 & 20,4 & 0,76 \\
\hline & & & & & \\
\hline & \multirow{2}{*}{\begin{tabular}{|l|} 
La Gouraudiaire \\
\end{tabular}} & maximum & 45 & 23,9 & 1 \\
\hline & & palier & 40 & 20 & 0,84 \\
\hline
\end{tabular}

\begin{tabular}{|c|c|c|c|c|c|}
\hline & & & & & \\
\hline \multirow{5}{*}{ SEC } & \multirow{2}{*}{ Cusset } & maximum & $\frac{\Phi}{48.2}$ & - cil kra & Tg Phy \\
\hline & & palier & 46,5 & 32,4 & $\frac{1,22}{1,05}$ \\
\hline & & & & & \\
\hline & \multirow[t]{2}{*}{ La Gouraudiaire } & maximum & 49,4 & 43,7 & 1,17 \\
\hline & & palier & 46,9 & 28,7 & 1,07 \\
\hline
\end{tabular}

3) Condition compacté au Kango

\begin{tabular}{|c|c|c|c|c|c|}
\hline & Site d'origine & & $\Phi$ en $^{\circ}$ & $\mathbf{C}$ en $\mathrm{kPa}$ & $\mathrm{Tg} \mathrm{Phy}$ \\
\hline \multirow{5}{*}{ SEC } & \multirow[t]{2}{*}{ Cusset } & maximum & 51,2 & 54,4 & 1,24 \\
\hline & & palier & 45,8 & 43,7 & 1,03 \\
\hline & & & & & \\
\hline & \multirow[t]{2}{*}{ La Gouraudiaire } & maximum & 47 & 66,3 & 1,07 \\
\hline & & palier & 45,4 & 47,4 & 1,01 \\
\hline \multirow{5}{*}{ SATURÉ } & \multirow[t]{3}{*}{ Cusset } & maximum & 49,4 & 19,8 & 1,17 \\
\hline & & palier & 45,2 & 56,5 & 1,01 \\
\hline & & & & & \\
\hline & \multirow[t]{2}{*}{ La Gouraudiaire } & maximum & 48,4 & 25,4 & 1,12 \\
\hline & & palier & 43,4 & 18,7 & 0,95 \\
\hline
\end{tabular}

Les courbes contraintes-déformations ont ensuite été interprétées dans le diagramme de Mohr pour en déduire les propriétés de résistance au cisaillement $C$ et $\tan \Phi$. Les résultats sont synthétisés sur le Tableau 1.

L'analyse des résultats fait apparaître les principales informations suivantes que nous pressentions de façon qualitative.

Tout d'abord, nous observons logiquement une augmentation des caractéristiques mécaniques suivant que le matériau est conditionné de façon foisonné, agencé ou compacté. Ainsi pour les matériaux du Cusset en condition non saturée, nous obtenons respectivement les valeurs de pic de $41,5^{\circ}, 48,2^{\circ}$ et $51,2^{\circ}$. Pour le matériau de la Gouraudiaire plus friable que celui du Cusset du fait de la minéralogie différente du ballast, l'opération de compactage vibratoire a modifié sa granulométrie faisant apparaître des matériaux plus fins, ce qui se traduit par des valeurs d'angle de frottement plus faibles pour le conditionnement compacté que pour le conditionnement agencé.

Les résultats montrent également que les propriétés de résistance au cisaillement $(C$ et tan $\Phi)$ sont globalement meilleures en condition non saturée qu'en condition saturée. Logiquement la condition saturée conduit à une lubrification des ballasts diminuant ainsi la résistance au cisaillement de ces matériaux. Ce comportement est analogue au comportement hydromécanique des enrochements de barrage (Bonelli et Anthiniac, 2000 ; Bonelli et al., 2000).

Ensuite, nous observons que les tassements restent proches entre les essais saturés et les essais non saturés ( $c f$. Fig. 12 relative au matériau du Cusset pour une pression de confinement de 100 et $400 \mathrm{kPa}$ ). Le fait que le matériau soit saturé ou pas influence peu les déformations macroscopiques lors du cisaillement et ceci quel que soit la contrainte de confinement appliquée.

Enfin, les essais de cisaillement mettent en évidence les phénomènes de contractance et de dilatance des ballasts. Les ballasts en conditionnement foisonné se contractent uniquement pendant le cisaillement et le volume n'augmente pas durant l'ensemble du déplacement. A contrario, les ballasts en conditionnement agencé ou compacté, après une phase de contractance en début d'essai, voient diminuer leur masse volumique suite à une dilatance marquée en deuxième partie d'essai (Fig. 13).

Ce programme de recherche a conduit à une difficulté expérimentale. En effet, les matériaux granulaires utilisés par la SNCF pour les ballasts de ligne LGV ont une taille 


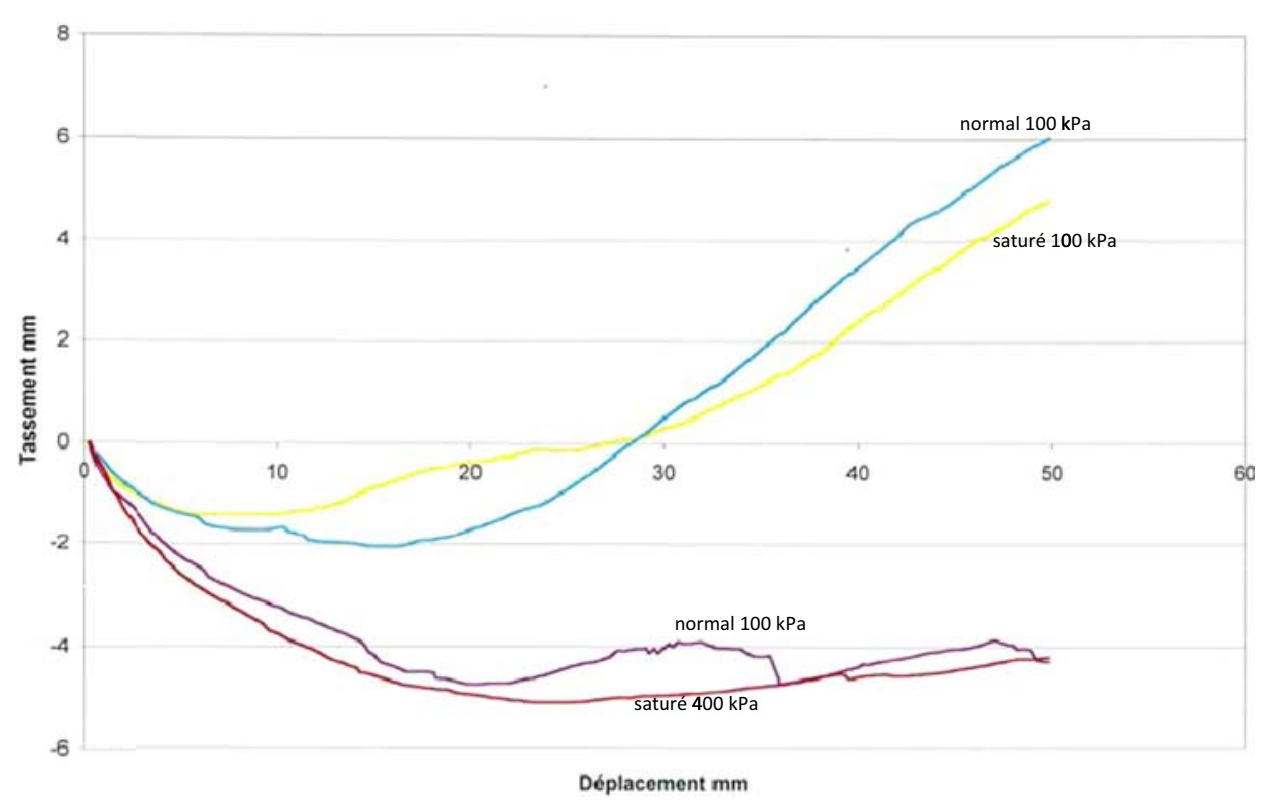

Fig. 12. Comparaison des tassements à sec et en saturé pour le matériau de Cusset.

Fig. 12. Comparison of dry settlements and saturated settlements for Cusset materials.

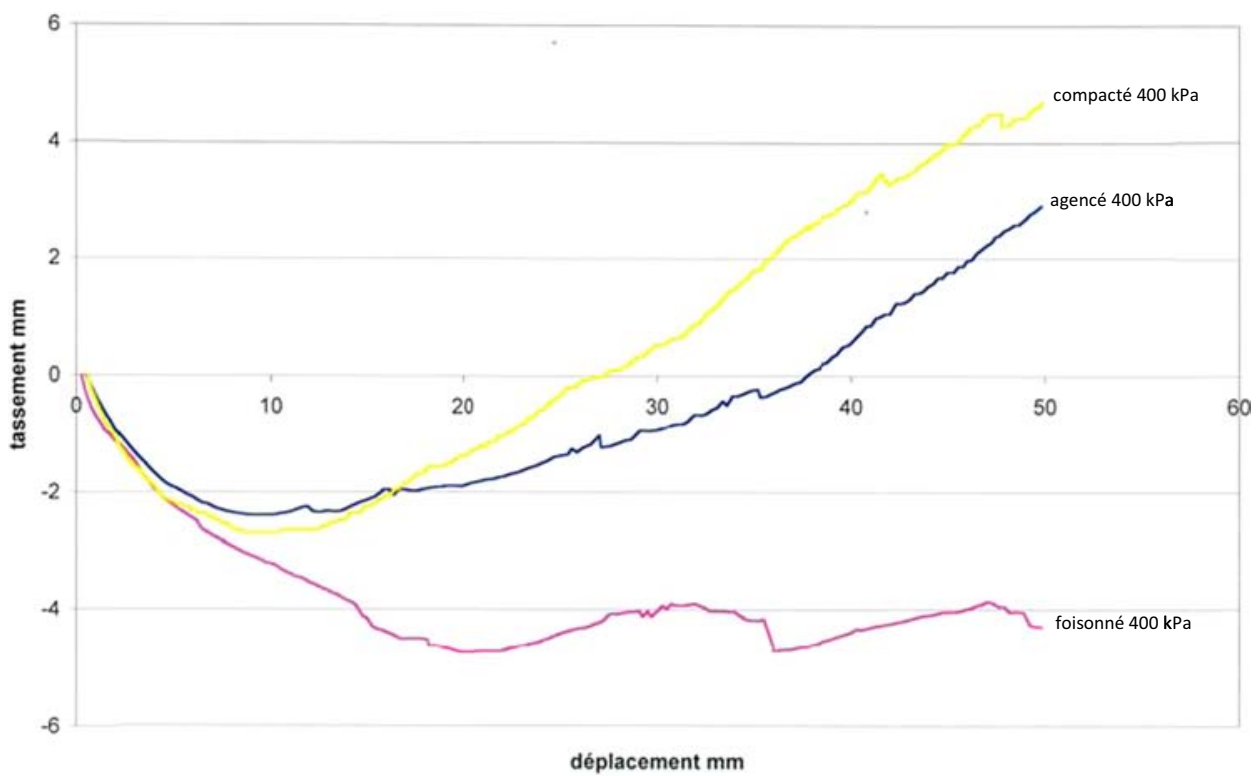

Fig. 13. Comparaison des tassements pour trois méthodes de compactage agencé-foisonné-compacté à $400 \mathrm{kPa}$ pour le matériau de Cusset. Fig. 13. Comparison of settlements for three different compaction methods.

granulométrique variant de 25 à $65 \mathrm{~mm}$ et ne contiennent aucune fine. Lors de la phase de cisaillement, d'importants phénomènes de dilatance sont mis en œuvre suite aux mouvements rhéologiques des grains. Ceci entraîne des forces de frottement latérales importantes au contact des parois du boîtier supérieur mobile et provoque un déplacement non négligeable tendant à le désolidariser du boîtier inférieur fixe.

La contrainte de cisaillement mesurée inclut la résistance au cisaillement propre des matériaux et une part, non mesurable mais que nous estimons faible, liée à la dilatance. La Figure 13 montre à ce titre une faible dilatance sous faibles contraintes normales.

\section{4 Étude des propriétés de résistance au cisaillement des interfaces entre géosynthétiques et sols}

\subsection{Contexte}

IRSTEA conduit une activité de recherche et d'expertise dans les domaines du comportement des produits géosynthétiques utilisés dans le Génie Civil. À ce titre, le laboratoire de géomécanique a étudié, à l'aide de la grande boîte de Casagrande, le comportement au cisaillement des interfaces entre un géotextile, une géomembrane ou plus largement un dispositif 


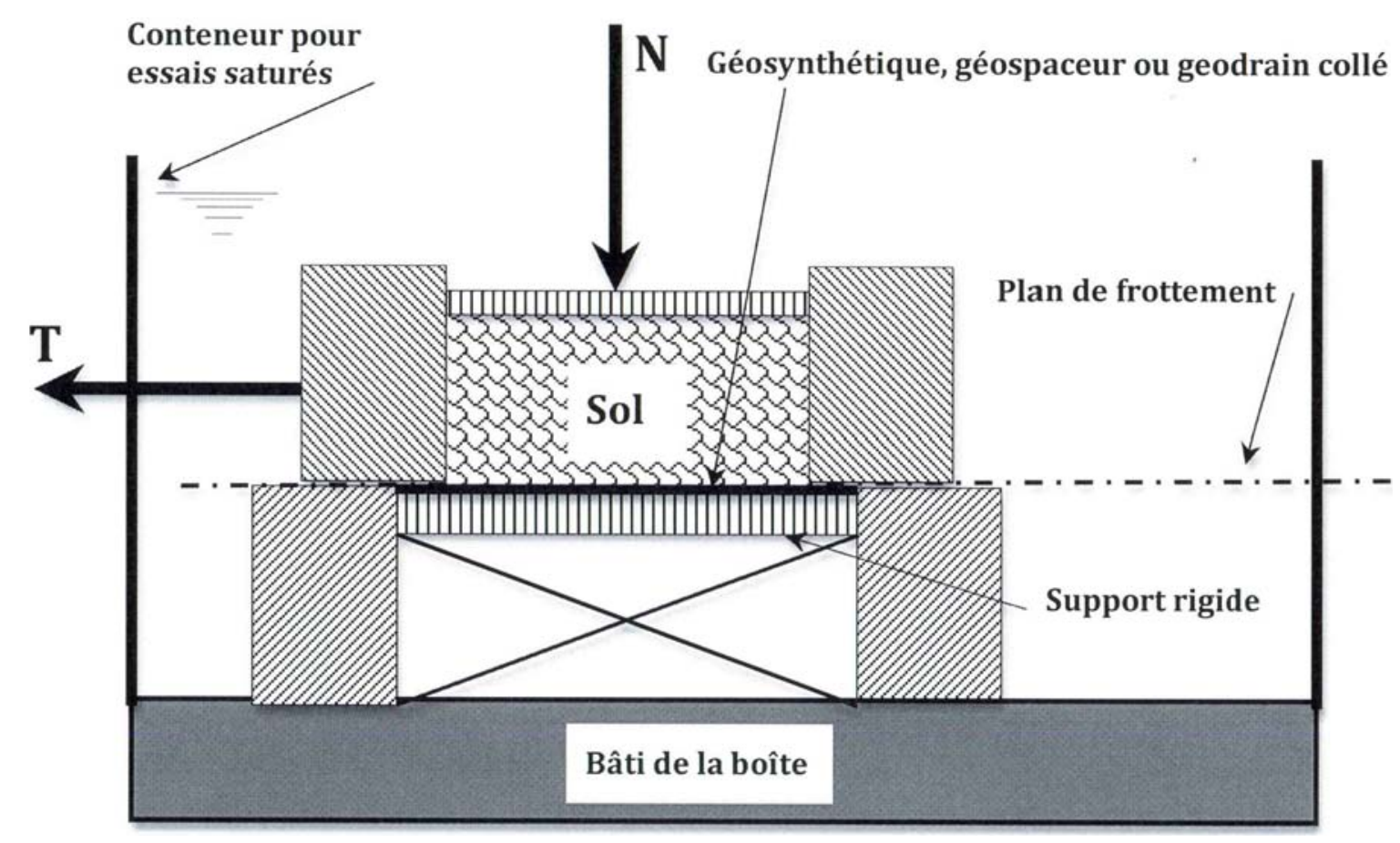

Fig. 14. Schéma de principe du montage permettant l'évaluation de l'effort de frottement à l'interface sol-géosynthétique.

Fig. 14. Schematic diagram to evaluate the friction force at the soil-geosynthetic interface.

d'étanchéité par géomembrane (DEG) et une structure support constitué d'un sol. Ces études ont pour objectif de déterminer les caractéristiques mécaniques des matériaux géosynthétiques, mais aussi la connaissance des lois de comportement et d'interface solgéosynthétique. Ces caractéristiques de frottement et de déformation sont des éléments indispensables pour le dimensionnement des DEG placés sur pente, utilisés notamment sur des ouvrages hydrauliques (Tacnet et Gotteland, 1999).

Les propriétés spécifiques des géosynthétiques et leurs interactions avec le sol imposent une définition précise des sollicitations mises en jeu. Pour répondre à cette demande, une adaptation a été mise en place sur la grande boîte de Casagrande afin de pouvoir réaliser des essais de frottement sur les géosynthétiques.

\subsection{Description du mode opératoire des essais types sur géosynthétiques à la grande boîte de Casagrande}

Nous proposons deux méthodes expérimentales destinées à séparer les efforts de frottement et les efforts d'ancrage :

- pour apprécier les efforts de frottement, nous collons la nappe de géosynthétique sur un support rigide, amovible, placé dans le demi-boîtier inférieur ou supérieur, le sol ou le géosynthétique d'interface se trouvant dans le demi-boîtier opposé (Fig. 14);

- pour apprécier les efforts d'ancrage, nous posons la nappe de géosynthétique sur une plaque d'appui en bronze fritté et nous l'ancrons à son extrémité (Fig. 15).

L'adaptation sur la grande boîte de Casagrande destinée à tester les géosynthétiques permet de faire des essais dans des conditions sèches ou saturées, avec un temps de mise en œuvre réduit, pour une configuration simple comme dans le cas d'une étude d'interface sol/géosynthétique classique ou pour des cas plus complexes comme pour l'étude d'un ensemble DEG comportant à la fois la géomembrane, le géocomposite drainant ou géoespaceur et le géotextile de protection.

\subsection{Exemple d'une étude visant à caractériser l'interface entre géosynthétique et sol conduite à partir de la grande boîte de Casagrande}

Nous avons conduit une étude pour le compte d'un producteur de géosynthétique visant à caractériser un géocomposite, constitué en partie centrale d'une géomembrane en PEHD (PolyÉthylène Haute Densité) de 1,5 mm d'épaisseur, recouverte par collage de part et d'autre de ses faces d'un géotextile aiguilleté en polypropylène de $4 \mathrm{~mm}$ d'épaisseur (Fig. 16).

La caractérisation mécanique de ce géocomposite consiste à déterminer les capacités frottantes de l'ensemble géotextile/géomembrane. Le matériau testé mis au contact du géocomposite est une grave 10/20. Quatre essais sont réalisés à des contraintes de confinement de $10,20,40$ et $60 \mathrm{kPa}$, en condition sèche et en condition humide.

Les résultats obtenus sont représentés aux deux figures suivantes (Fig. 17 et 18).

Nous constatons un angle de frottement élevé entre la grave et la partie aiguilletée du géocomposite. La réalisation de l'essai en condition humide permet de mettre en évidence une perte de résistance au frottement de l'ordre de 2 degrés environ (Tab. 2).

Ces résultats d'essais doivent toujours être interprétés de plusieurs façons, à charge pour le concepteur de 


\section{Essai de frottement sol/nappe posée sur plaque de bronze ancrée}

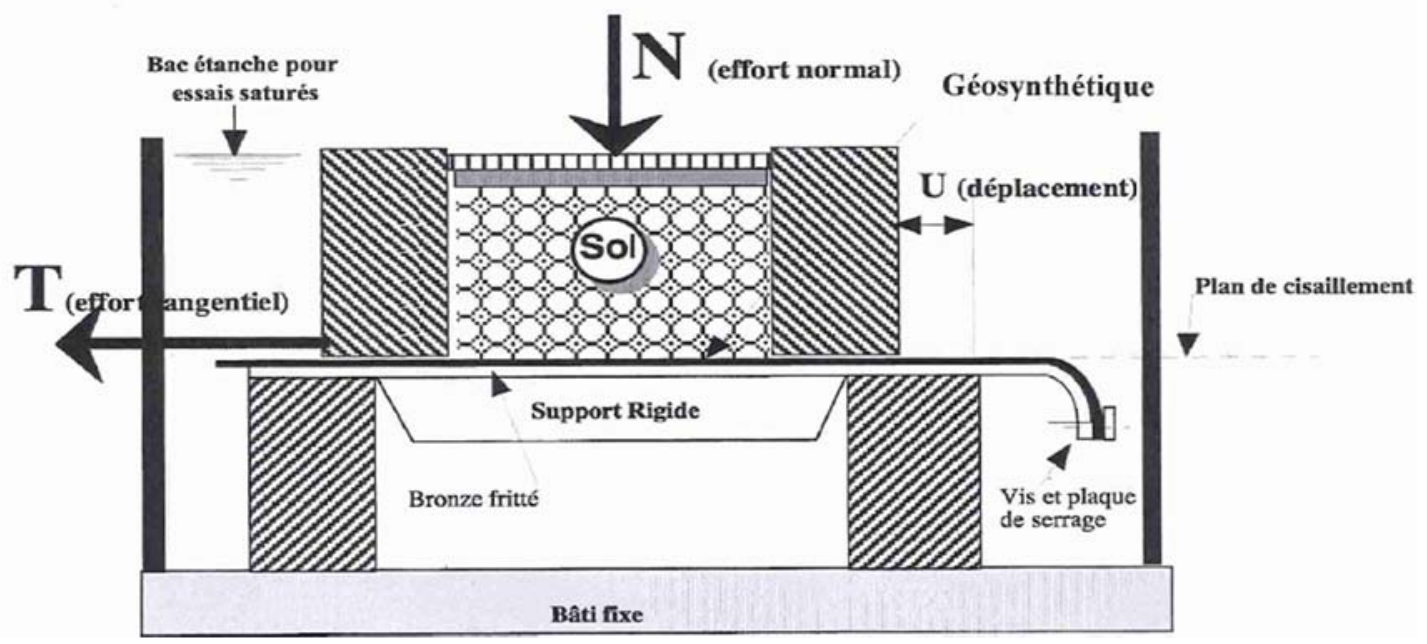

Fig. 15. Schéma de principe du montage pour l'évaluation de l'effort d'ancrage sol/géosynthétique.

Fig. 15. Schematic diagram to evaluate the soil/geosynthetic anchor force.

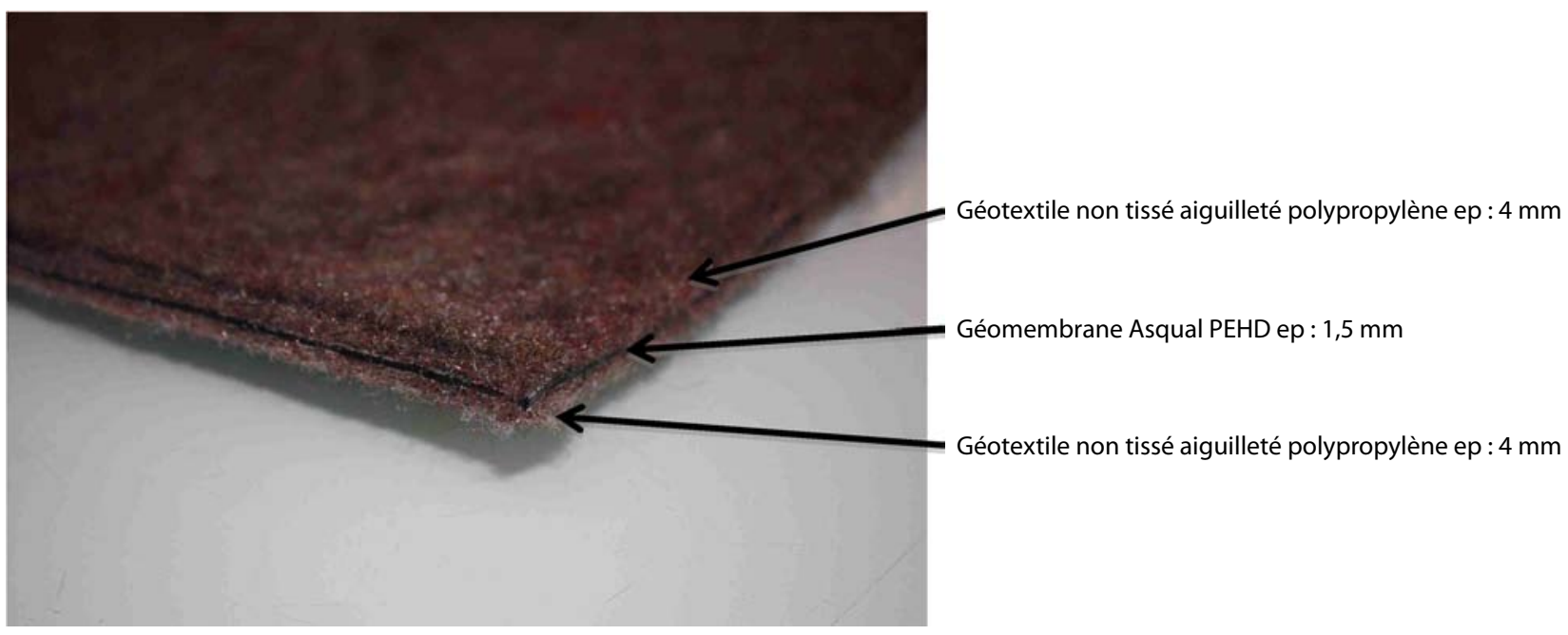

Fig. 16. Géocomposite constitué d'une géomembrane PEHD et de deux géotextiles aiguilletés en polypropylène.

Fig. 16. Geocomposite consisting of a HDPE geomembrane and of two needle-punched polypropylene geotextiles.

retenir la plus pertinente pour l'ouvrage à réaliser ou à vérifier :

- à la rupture, en considérant les contraintes de pic ;

- en grandes déformations, ce qui donne accès aux caractéristiques résiduelles ;

- mais aussi en petites déformations ( $1 \%$ de déformation par exemple), ce qui sera, le plus souvent, le critère à retenir pour le dimensionnement d'un ouvrage pour lequel les déformations doivent rester faibles, au risque d'endommager la barrière étanche.

Une limite de cette recherche expérimentale sur les géosynthétiques impliquant la grande boîte de Casagrande est liée aux faibles résistances au cisaillement des interfaces solgéosynthétiques, notamment lorsqu'elles sont appréciées sous faibles contraintes normales telles que cela est constaté sur les ouvrages réels impliquant des géosynthétiques. Dans ces conditions, la contrainte de confinement est appliquée par l'intermédiaire de masses car la précision de l'asservissement hydraulique fournie pas les vérins s'avère insuffisante pour les faibles contraintes allant de 0 à $15 \mathrm{kPa}$.

\section{5 Étude de la résistance au cisaillement des joints de reprise de bétonnage des Bétons Compactés au Rouleau (BCR)}

\subsection{Contexte}

Depuis 2008, le laboratoire de géomécanique d'IRSTEA conduit régulièrement des études de la résistance au 
Irstea d'Aix en Provence
Essais de frottement sol/géocomposite à la boîte de $30 \times 30 \mathrm{~cm} 2$ suivant norme NF P 84-505 modifiée selon annexe SNCF IN 0261 Géocomposite testé à sec

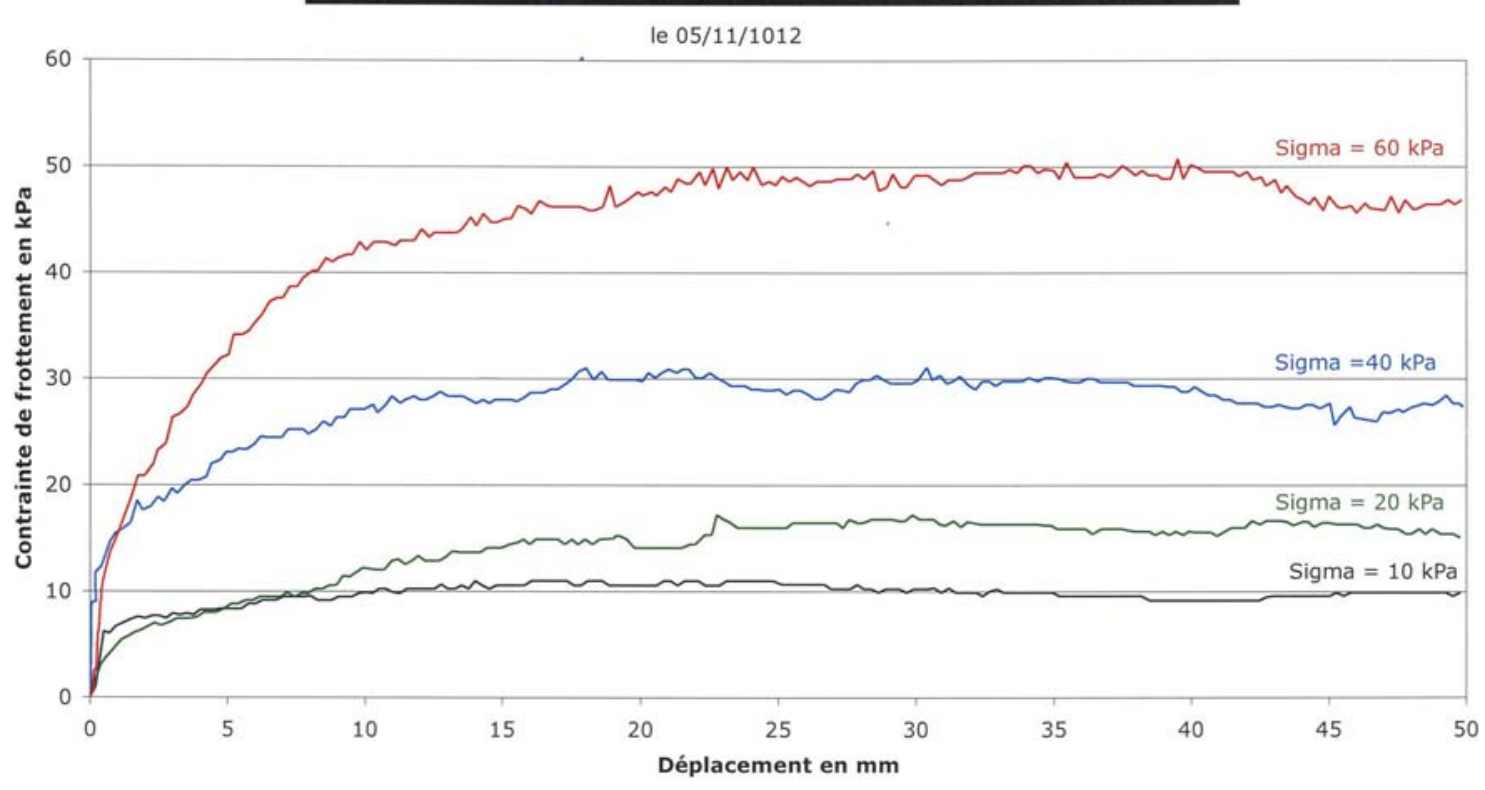

Fig. 17. Courbes contraintes/déplacements.

Fig. 17. Stress/displacement curves.

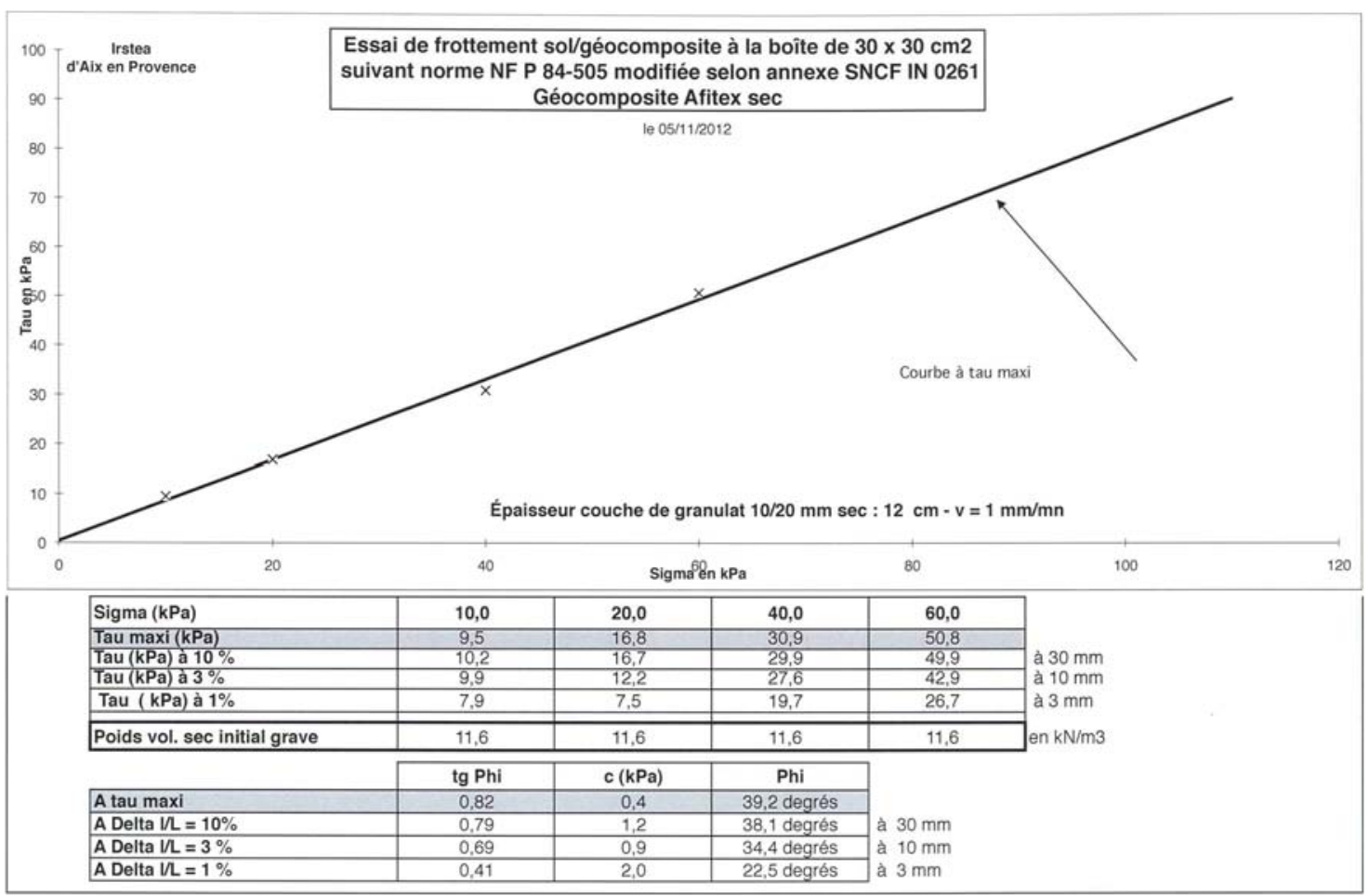

Fig. 18. Droite de Coulomb et tableau de valeurs associé.

Fig. 18. Coulomb model and the table of associated values. 
Tableau 2. Résultats des essais de frottement sur le géocomposite.

Table 2. Results of the friction tests on the geocomposite.

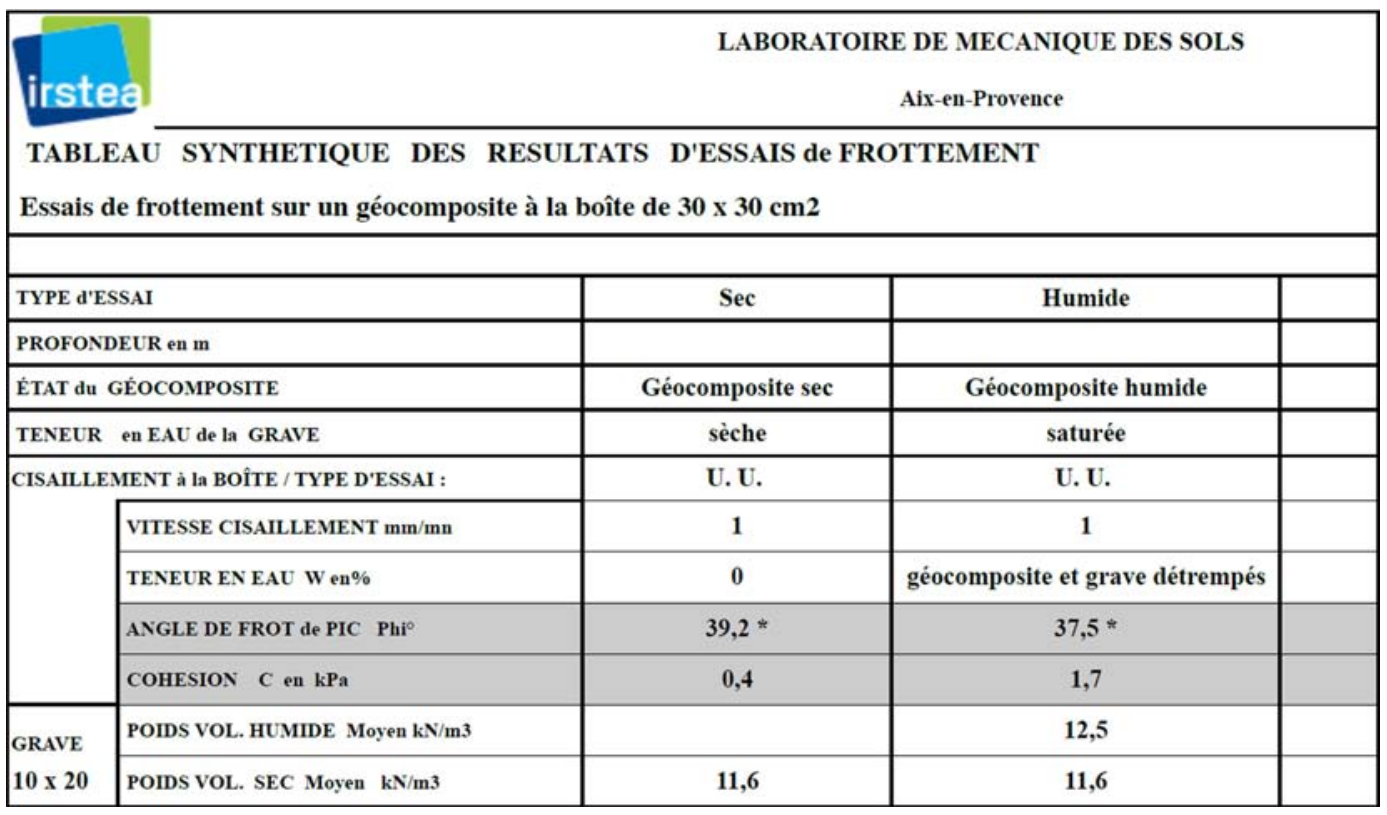

cisaillement des joints de reprise de bétonnage des Bétons Compactés au Rouleau (BCR) dans le cadre de projet de conception et de réalisation de barrages poids en BCR. Cette implication d'IRSTEA dans l'étude des barrages en BCR est issue de sa contribution initiale au projet national BACARA relatif au béton compacté au rouleau (BaCaRa, 1996).

Dans ce cadre, plusieurs études impliquant des essais de cisaillement sur des éprouvettes en béton BCR de grande dimension ont été conduites :

- le projet du barrage de Lurberria à St Pée de Nivelle en France (2007) ;

- le projet du barrage de Boussiaba situé dans la région de Jijel en Algérie (2008) ;

- le projet de barrage EdF de Rizzanèze en Corse (2011).

Les essais réalisés ont pour objectif la détermination de la résistance au cisaillement des joints de reprise de bétonnage du BCR, que l'on traduit classiquement par une valeur de cohésion et d'angle de frottement. Ces caractéristiques sont obtenues par l'intermédiaire d'essais de cisaillement à la grande boîte de Casagrande de $50 \times 50 \mathrm{~cm}^{2}$, réalisés sur des carottes cylindriques ayant un diamètre variant de 150 à $250 \mathrm{~mm}$ et contenant des granulats compris entre 40 à $63 \mathrm{~mm}$.

\subsection{Méthode expérimentale et adaptation du dispositif de cisaillement pour les éprouvettes cylindriques}

Afin de réaliser des essais sur des carottes de BCR, nous avons développé un appareillage expérimental permettant de réaliser le cisaillement d'éprouvettes cylindrique en béton de
$250 \mathrm{~mm}$ de diamètre tout en s'adaptant à la technologie du boîtier 50 par $50 \mathrm{~cm}^{2}$ de la grande boîte de Casagrande (Fig. 19).

Les éprouvettes de béton sont insérées dans les fourreaux guides de telle sorte à ce que le plan de cisaillement de la boîte coïncide avec le plan de reprise de bétonnage (Fig. 20). L'essai de cisaillement est ensuite réalisé à vitesse constante de l'ordre de $2 \mathrm{~mm} / \mathrm{min}$.

Les essais sont réalisés de façon analogue à ceux correspondant à l'essai classique de cisaillement. Ils consistent à mener plusieurs essais correspondant à différentes charges normales jusqu'à la rupture, sur des éprouvettes intactes issues du même joint et du même secteur du barrage.

\subsection{Résultats des essais de cisaillement sur les bétons BCR}

La courbe contrainte-déformation montre quatre phases caractéristiques distinctes (Fig. 21) :

- une phase d'adaptation (comprise entre 0 et $28 \mathrm{~mm}$ sur la Fig. 23) correspondant à la mise en place de l'éprouvette dans son boîtier ;

- une phase élastique (comprise entre $28 \mathrm{~mm}$ et $35 \mathrm{~mm}$ sur la Fig. 23) pendant laquelle on observe une montée en contrainte quasi linéaire ;

- une rupture fragile (autour de $37 \mathrm{~mm}$ sur la Fig. 23) pour laquelle on observe visuellement la rupture du joint et graphiquement une baisse instantanée de la résistance au cisaillement ;

- une phase de résistance résiduelle (au-delà de $37 \mathrm{~mm}$ sur la Fig. 23) correspondant au frottement des deux épontes obtenues après cisaillement de la carotte. 


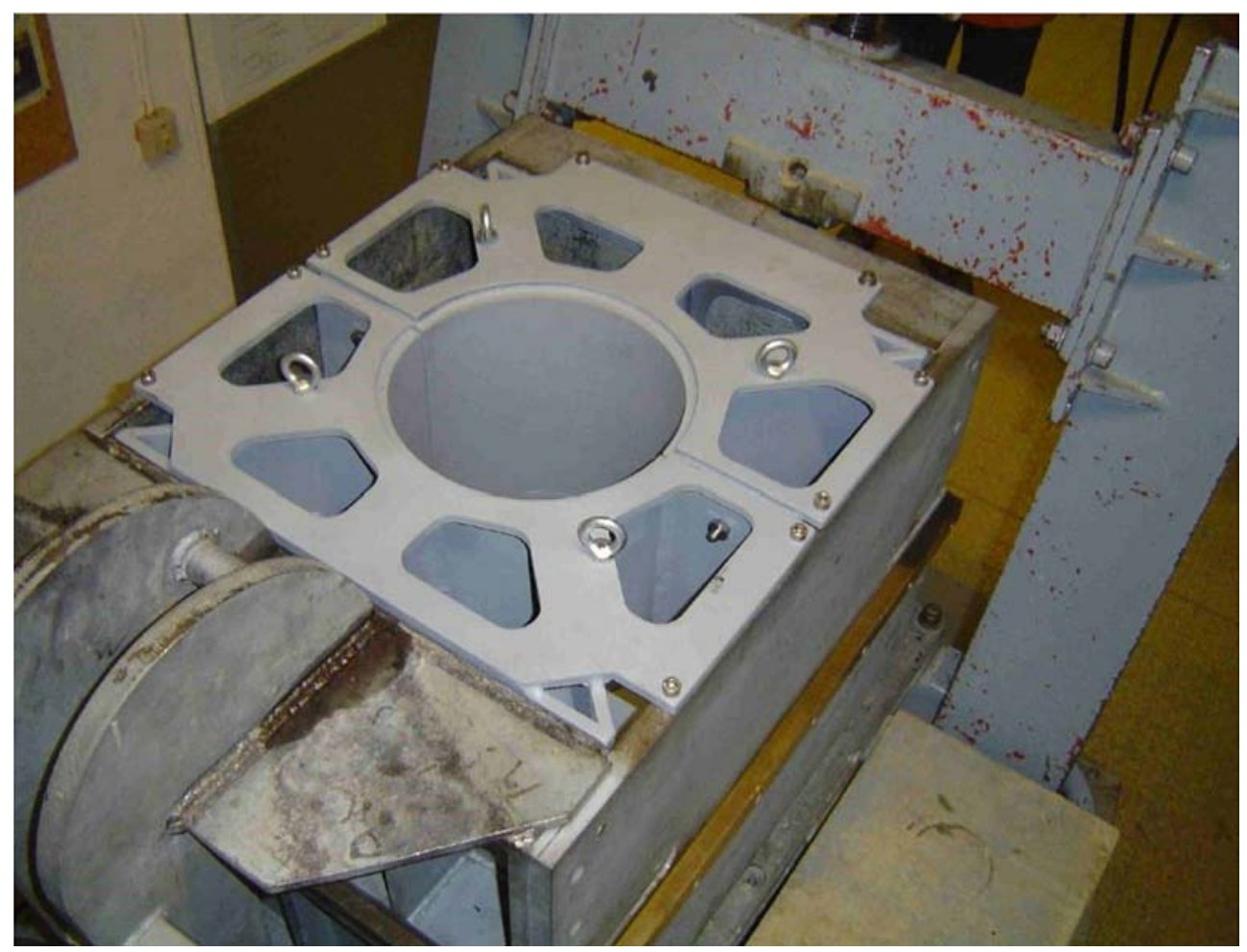

Fig. 19. Adaptateur inséré dans le boîtier supérieur de la grande boîte de Casagrande de $50 \times 50 \mathrm{~cm}^{2}$. Fig. 19. Adapter inserted into the upper half-housing of the large Casagrande box $50 \times 50 \mathrm{~cm}^{2}$.

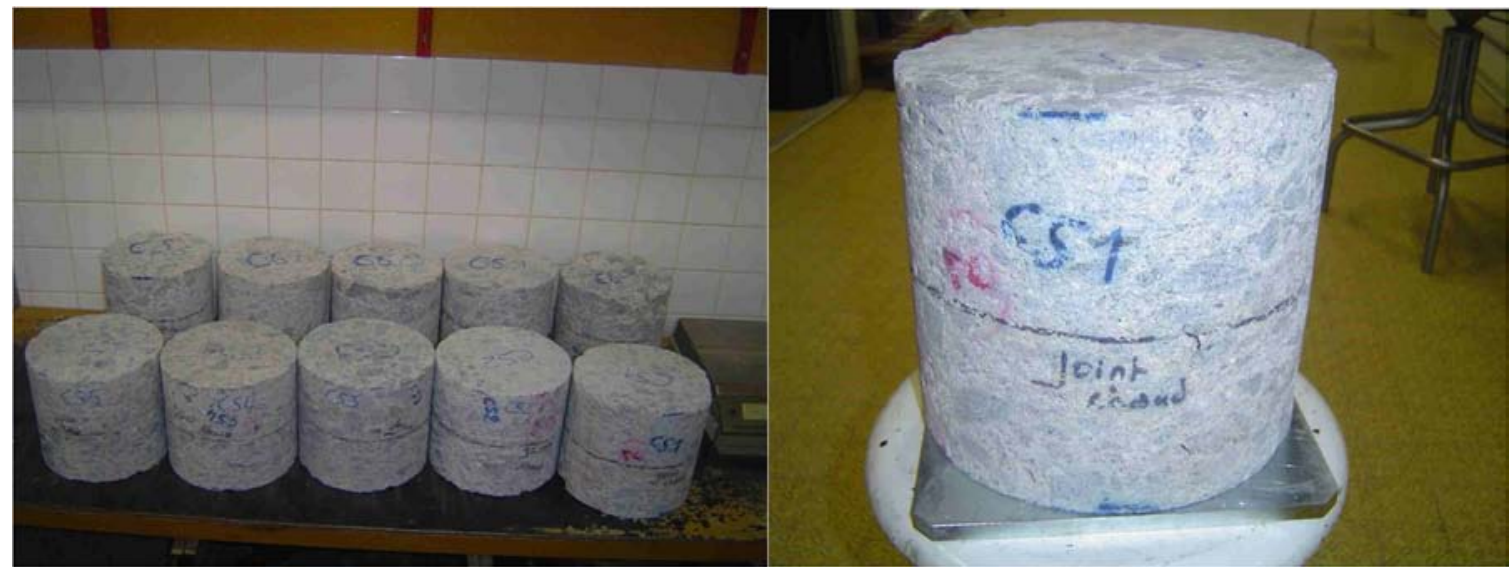

Fig. 20. Série d'éprouvettes béton BCR à tester (gauche) et éprouvette de BCR avec l'indication du plan de joint de reprise (droite). Fig. 20. Series of RCC concrete samples to be tested (left) and RCC sample with the indication of the recovery joint plane (right).

L'interprétation des essais permet d'obtenir la résistance maximale à la rupture de chaque éprouvette et la valeur du frottement résiduel (Fig. 22). Il est alors possible d'obtenir les valeurs caractéristiques $C$ et $\tan \Phi$ au moyen d'un graphe de Mohr-Coulomb à partir des différentes ruptures réalisées (Fig. 22).

Une limite de cette recherche expérimentale sur les bétons BCR impliquant la grande boîte de Casagrande peut être liée à la rigidité limitée de notre équipement. Elle reste suffisante pour traiter la question des joints de reprise de bétonnage des bétons $\mathrm{BCR}$, mais pourrait conduire à des difficultés si nous réalisions des essais de cisaillement sur des matériaux de grande résistance, tel que le béton de masse.

\section{6 Étude du renforcement des sols par la végétation}

\subsection{Contexte}

IRSTEA a été impliqué avec le CIRAD en 2011 dans le projet de recherche ANR ECOSFIX. Il s'agit d'un projet de recherche d'ingénierie écologique visant à étudier les 


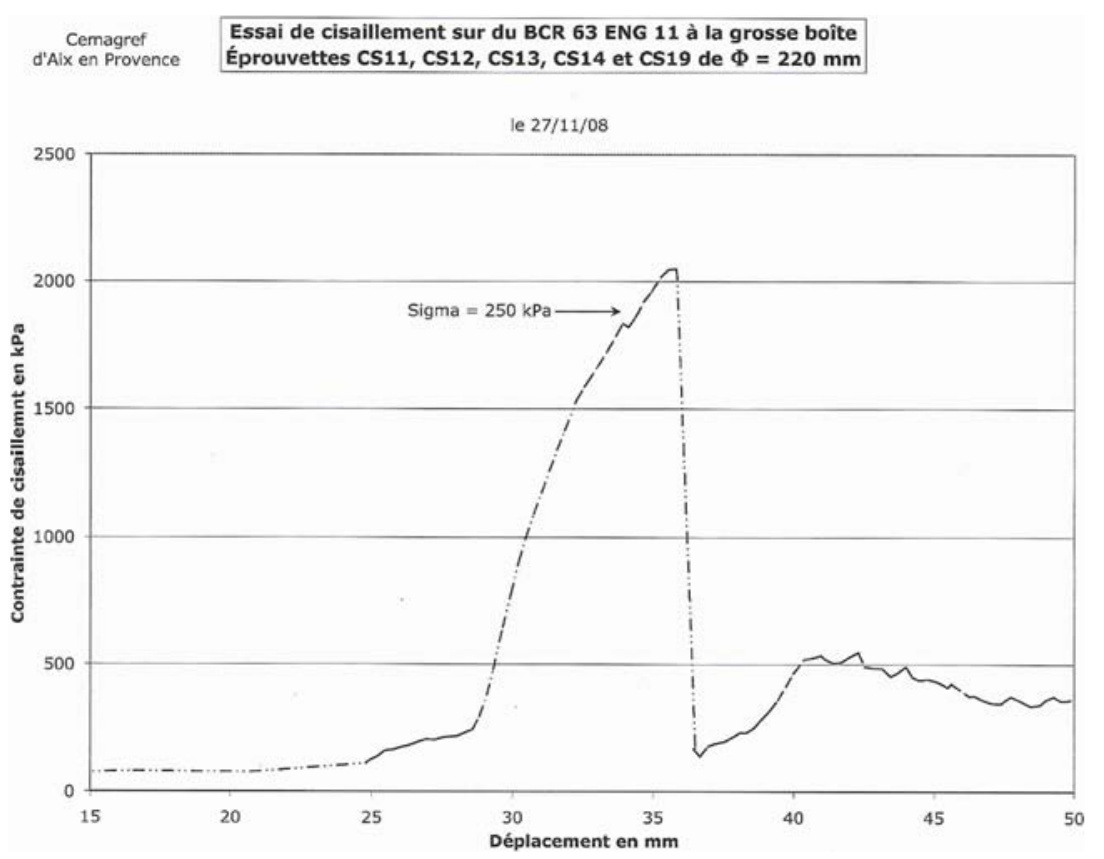

Fig. 21. Courbe contrainte de cisaillement en fonction du déplacement à $250 \mathrm{kPa}$.

Fig. 21. Shear stress vs displacement at $250 \mathrm{kPa}$.

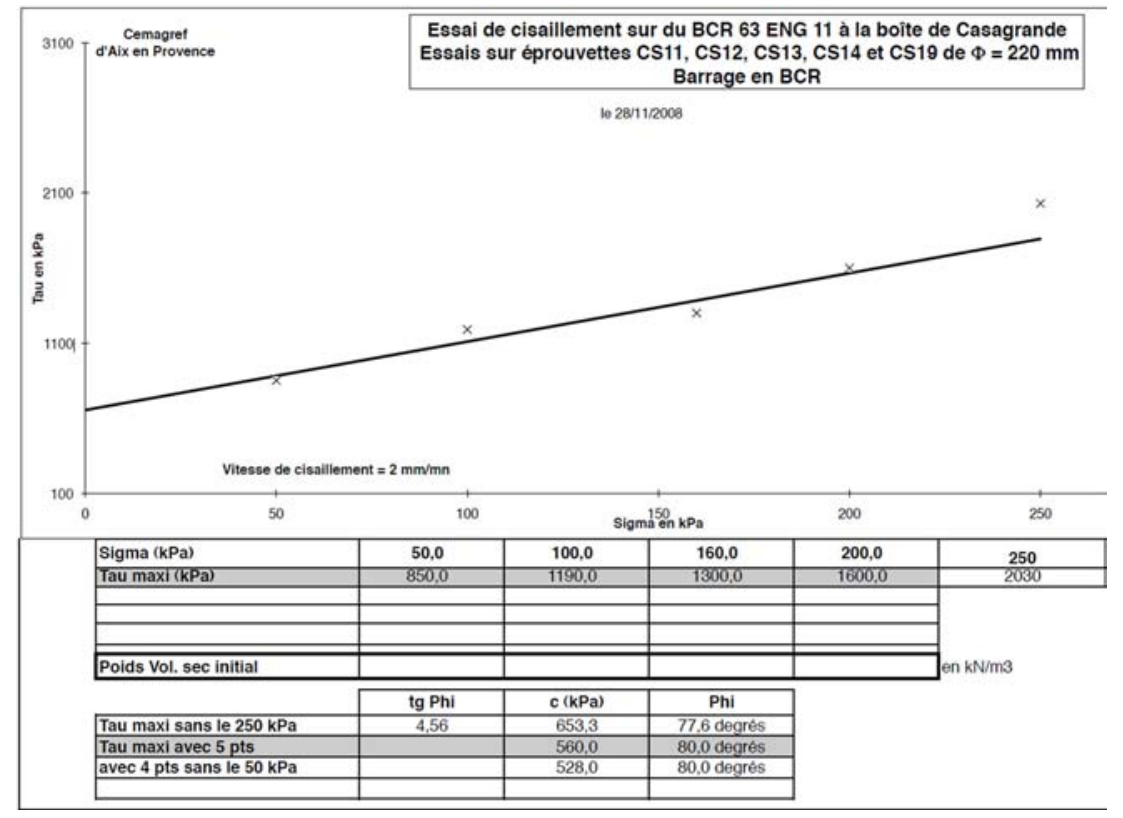

Fig. 22. Représentation en Mohr/Coulomb des résultats des essais de cisaillement.

Fig. 22. Representation of the shear test results in Mohr - Coulomb diagram.

glissements de terrain et les modalités par lesquelles la végétation peut stabiliser et réduire le risque de glissement des pentes instables (Ghestem et al., 2012).

La contribution du laboratoire de géomécanique d'IRSTEA dans ce projet est de vérifier si une amélioration de la résistance au cisaillement peut être apportée grâce à la présence de racines. L'objectif était de tester des végétaux en vraie grandeur au moyen du boîtier de $50 \times 50 \mathrm{~cm}^{2}$ de la grande boîte de Casagrande. La pertinence d'essais sur ce banc de cisaillement de grande dimension repose sur le fait que nous souhaitons évaluer l'effet de renforcement des systèmes racinaires complets. Étant donné les espèces testées, il fallait des dimensions de l'ordre de $50 \mathrm{~cm}$ de diamètre. L'autre aspect essentiel des essais conduits dans ce projet est d'évaluer les effets des traits architecturaux des systèmes racinaires, qualifiant la forme du système racinaire, pour le renforcement du sol afin de les intégrer dans les modèles de stabilité de pentes (Ghestem et al., 2012 ; Veylon et al., 2015). 


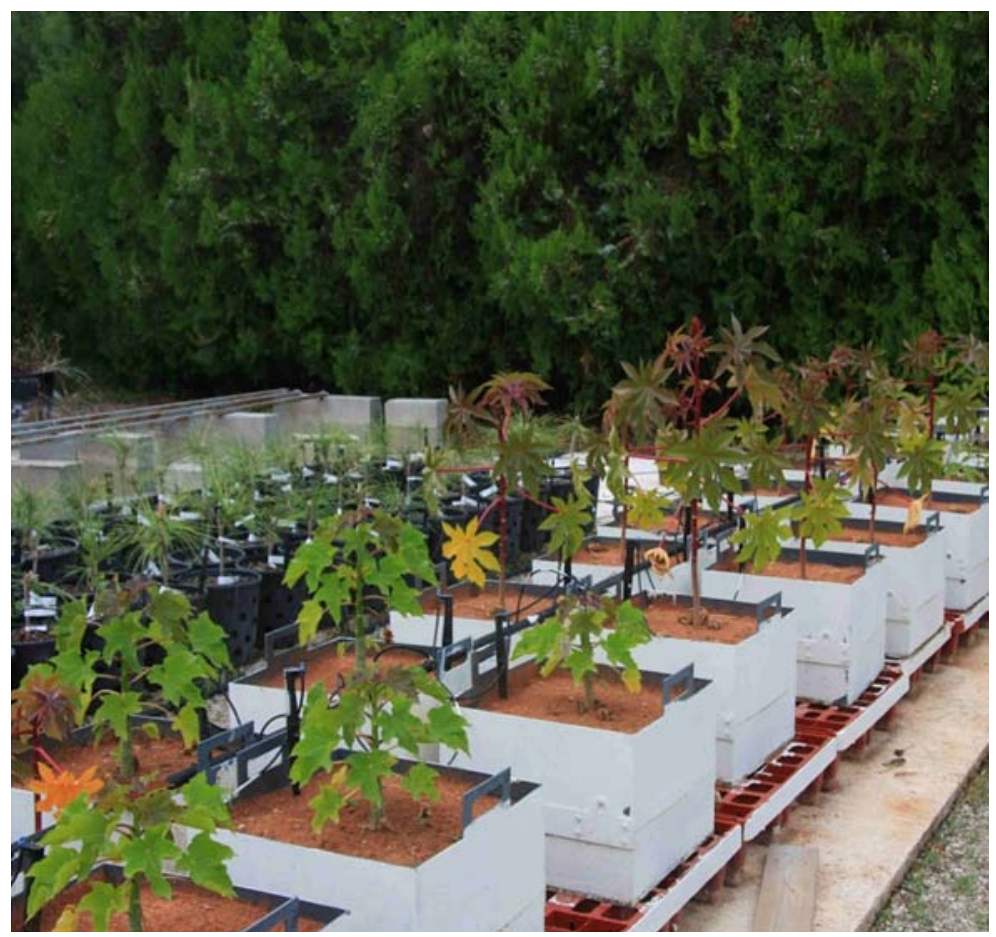

Fig. 23. Série d'arbustes en phase de développement en pépinière dans les boîtiers PVC de cisaillement.

Fig. 23. Shrubs nursery development phase in shear PVC housings.

La difficulté de cette étude est de reproduire les conditions de sol en surface, correspondant à des niveaux de contraintes normales très faibles.

\subsection{Méthode expérimentale et adaptation du dispositif de cisaillement à des fins d'essai sur des sols végétalisés}

L'étude a été réalisée à partir de trois espèces végétales originaires d'Asie, susceptibles de stabiliser de manière efficace les terrains en Chine (province du Yunnan). Ces trois espèces végétales ont été plantées dans des boîtiers PVC remplis d'un sol limoneux argileux répondant aux mêmes caractéristiques que le sol local. Elles ont connu alors une période de développement végétal et racinaire de six mois en pépinière (Fig. 23).

Les boîtiers PVC ont été conçus spécifiquement pour être testés à la grande boîte de Casagrande. Les deux demi-parties ont été solidarisées dans un premier temps pour permettre le déroulement normal de la phase de compactage et de saturation/consolidation. Elles ont été ensuite déconnectées lors de la mise en place dans les boîtiers de la grande boîte de Casagrande, pour permettre le cisaillement du sol dans les conditions normales de l'essai.

Pour reproduire les très faibles contraintes normales des sols de surface requises pour ces essais, nous avons utilisé des masses additionnelles en métal, l'asservissement hydraulique n'étant pas suffisamment précis pour de si basses contraintes. Les masses définies pour une contrainte normale donnée ont été positionnées sur la plaque d'application des contraintes et permettent ainsi de travailler même pour de très faibles valeurs de confinement des matériaux (de 0 à $10 \mathrm{kPa}$ ). Les tiges des arbustes ont été coupées à ras du boîtier de façon à pouvoir appliquer uniformément la force de confinement sur le plateau horizontal.

Par ailleurs, pour être le plus représentatif de la réalité des sols de surface, le matériau a été compacté de manière à être très peu dense $\left(\gamma_{\mathrm{d}}=12,8 \mathrm{kN} / \mathrm{m}^{3}\right.$ à $\left.\mathrm{w}=15 \%\right)$.

Pour ce type d'essais se déroulant sur une longue période, la phase de consolidation du sol a été un point délicat à traiter car il a nécessité un suivi précis du niveau d'hydratation dans le temps. Un contrôle de la teneur en eau a été effectué à partir de prélèvements réguliers de sol. Cette phase délicate a été maîtrisée grâce à l'installation d'un réseau de goutteurs en pépinière, gérant automatiquement l'hydratation des matériaux. Des prélèvements intacts ont été effectués dans la zone du plan de cisaillement après essai, afin de vérifier la densité et la teneur en eau du sol testé.

\subsection{Résultats obtenus}

Plusieurs essais sur un sol limoneux argileux, d'une part non renforcé (sol seul) et d'autre part renforcé par des végétaux, ont été effectués et ont donné les résultats suivants (Fig. 24).

Les différentes formes de courbes obtenues pour un même niveau de pression de confinement et pour une même espèce végétale (Fig. 25) sont liées au caractère très peu dense du matériau mis en place, posant des difficultés d'ordre expérimental sur la répétabilité des essais.

Malgré ces inconvénients et si on compare les résultats avec ou sans végétation, les essais réalisés ont montré un accroissement de la résistance au cisaillement (léger pour deux espèces et plus sensible pour une autre espèce). 
Cemagref d'Aix en Provence
Essais de cisaillement à la grosse boîte de $50 \times 50 \mathrm{~cm} 2$ Échantillon de sol limoneux-argileux du site du Cemagref d'Aix Boîtiers PVC n A, B, 17 et 19

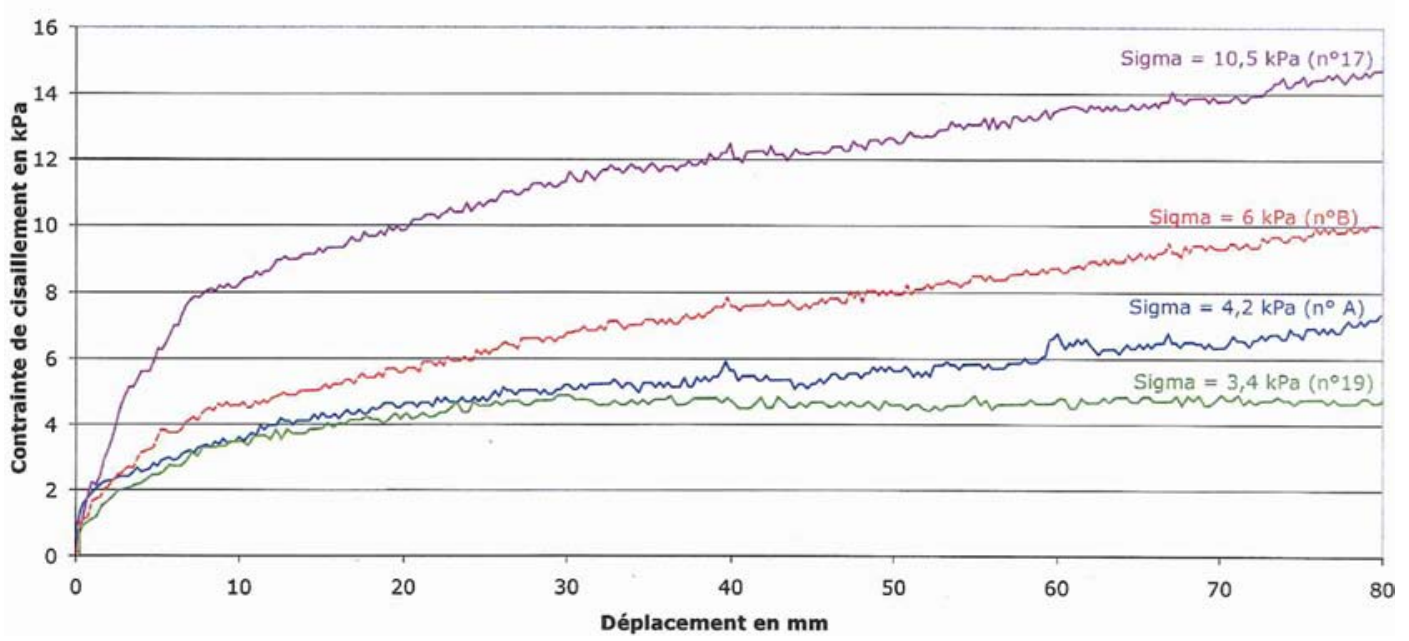

Fig. 24. Courbes contrainte-déformation du sol limoneux argileux sans racines.

Fig. 24. Stress-strain curves for silty clay soil without roots.

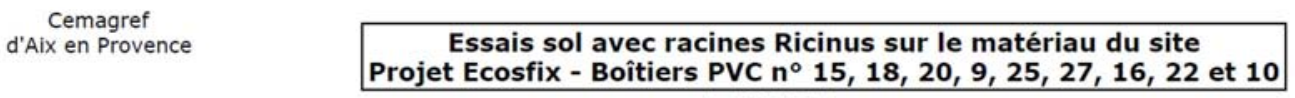

le $14 / 03 / 2011$

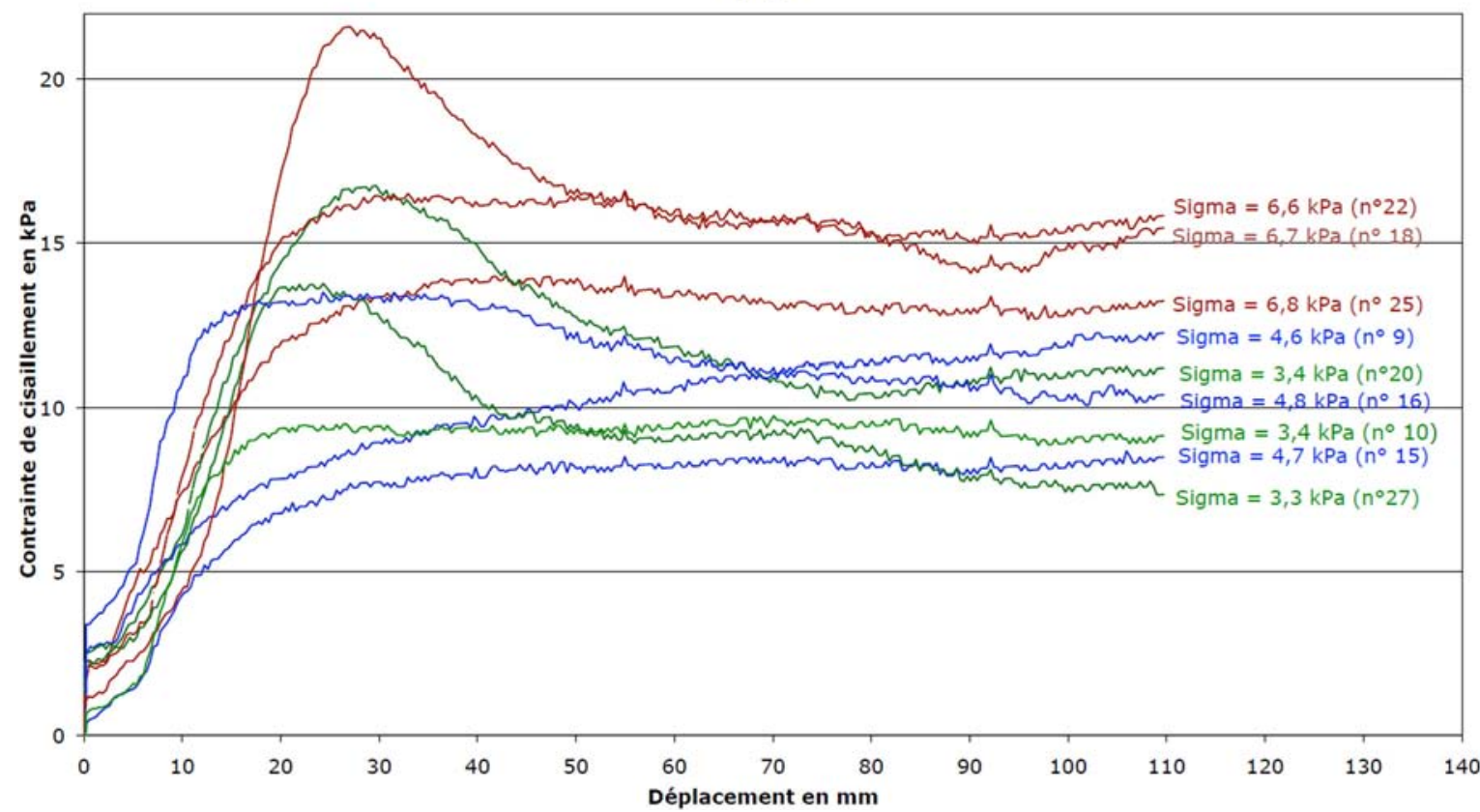

Fig. 25. Courbes contrainte-déformation du sol limoneux argileux avec arbuste Rhicinus.

Fig. 25. Stress-strain curves of the silty clay soil with Ricinus shrub.

Avec l'adoption du choix technique de bacs PVC préparés à distance du laboratoire et intégrés a posteriori dans la grande boîte de Casagrande pour les essais de cisaillement, il a été démontré qu'il était possible de travailler sur des sols limoneux argileux renforcés.

Une limite de cette recherche expérimentale impliquant la grande boîte de Casagrande est liée à la réalisation des essais sous très faibles contraintes normales. En effet, il est apparu délicat de discriminer les résultats correspondant à différentes contraintes normales de faibles valeurs absolues. Dans ces conditions, nous sommes proches des limites de l'utilisation de la grande boîte de Casagrande, du fait de l'inertie de l'équipement. Nous renvoyons les lecteurs intéressés vers les références Ghestem et al. (2012) et Veylon et al. (2015) qui 
développent respectivement le rôle de l'architecture racinaire dans la résistance au cisaillement des sols et la quantification des propriétés mécaniques et hydriques du sol liées aux racines.

\section{Conclusions}

Cet article montre que la grande boîte de Casagrande est un appareil aux multiples applications, que se soit pour des essais normalisés ou pour des essais en recherche et développement, moyennant des adaptations et des évolutions originales.

La représentativité des résultats des essais de laboratoire par rapport aux matériaux réels mis en place sur les ouvrages a été une préoccupation constante qui a conduit à faire des essais en testant l'évolution des caractéristiques mécaniques en fonction de l'évolution de la granulométrie. Ceci explique le développement d'un appareil de cisaillement de $30 \times 30 \mathrm{~cm}^{2}$ de section, puis le passage d'un boîtier de $30 \times 30 \mathrm{~cm}^{2}$ de section vers un boîtier de $50 \times 50 \mathrm{~cm}^{2}$, afin de caractériser des matériaux pouvant contenir des grains jusqu'à $80 \mathrm{~mm}$.

Conçu à l'origine pour étudier les sols grossiers des barrages à la fin des années 1970, ce banc d'essai a été adapté à des projets de recherche spécifiques aux sols grossiers. L'étude du comportement des ballasts SNCF des lignes LGV présentée dans l'article illustre ce point. Par ailleurs, l'asservissement hydraulique et le pilotage par ordinateur ont permis de réaliser des essais en utilisant uniquement la fonction de confinement, à l'instar d'un banc œdomètre. Une recherche remarquable dans ce cadre a été l'étude du comportement hydromécanique des enrochements soumis au phénomène d'imbibition.

Des adaptations nouvelles de la grande boîte de Casagrande ont également permis de contribuer au projet national BACARA et de répondre à des sollicitations concernant des barrages en $\mathrm{BCR}$ au moyen d'études visant à caractériser les performances des joints de reprise de bétonnage des bétons BCR. Plus récemment, d'autres adaptations ont permis de conduire des essais dans le projet ANR ECOSFIX visant à évaluer le rôle des racines dans la résistance au cisaillement d'un sol de surface soumis à faibles contraintes normales.

Enfin, l'utilisation de la grande boîte de Casagrande pour la détermination des comportements aux interfaces des géosynthétiques et la participation à l'élaboration des normes sur les mêmes géosynthétiques ont favorisé l'expertise sur la connaissance et l'utilisation de ces matériaux dans les ouvrages hydrauliques de génie civil.

\section{Références}

BaCaRa. 1996. Le Béton Compacté au Rouleau - Les barrages en BCR. Presses Ponts et Chaussées, Paris (France), 200 p.

Bonelli S, Anthiniac P. 2000. Modélisation hydroplastique du premier remplissage d'un barrage en enrochements. In: Proc. 53 ème Conférence Canadienne de Géotechnique, Montréal (Canada), pp. 255-262.

Bonelli S, Anthiniac P, Bernard A. 2000. Comportement hydromécanique des enrochements de barrage. In: Proc. Colloque physique et mécanique des matériaux granulaires, Université de Marne la Vallée (France), pp. 73-78.

Bourdeau Y. 1997. Le comportement des alluvions du Rhône dans une grande boîte de cisaillement direct. Rev Fr Geotech 79: 45-57.

CIGB. 1993. Commission Internationale des Grands Barrages. Matériaux rocheux pour barrages en enrochement. Synthèse et recommandations. Bulletin 92. Icold Ed. Paris, 132 p.

Ghestem M, Veylon G, Stokes A, Bernard A. 2012. Influence of plant root system morphology and architectural traits on soil shear resistance. Plant Soil, DOI:10.1007/s11104-012-1572-1

Gotteland P, Tacnet JM, Aboura A. 2000. Caractérisation des sols grossiers - Application aux sols de torrents. Rev Fr Gen Civ 4(7-8): 325-338.

Manba M. 1989. Résistance au cisaillement des enrochements et sols grossiers. Application aux calculs de barrage. Thèse de doctorat à l'Université de Lille I (France).

Marsal RJ, Fuentes de la Rosa A. 1976. Mechanical Properties of Rockfill Soil Mixtures. In: ICOLD $12^{\text {th }}$ International Congress on Large Dams, Mexico, March 29 - April 2, 1976, pp. 179-209.

Pedro L. 2004. Étude du comportement mécanique de sols hétérogènes modèles à son application au cas des sols naturels. Thèse de doctorat, École Nationale des Ponts et Chaussées (France), 294 p.

Perrot A. 1968. Étude de la résistance au cisaillement des matériaux grossiers. Bulletin 133, Rapport du Laboratoire des Ponts et Chaussées, Nancy (France), 40 p.

Serre D, Peyras L, Curt C, Boissier D, Diab Y. 2007. Évaluation des ouvrages hydrauliques de génie civil. Can Geotech $J$ 44: $1298-1313$

Tacnet JM, Gotteland P. 1999. Sols grossiers renforces pour ouvrages en sites instables. In: Actes du colloque Rencontres géosynthétiques 99, Bordeaux (France), Vol. 2. pp. 111-118.

Tacnet JM, Gotteland P, Bernard A, Mathieu G, Deymier C. 2000. Mesure des caractéristiques géotechniques des sols grossiers Application aux sols de torrent. In: $4^{\text {eme }}$ Colloque International InterPraevent, Villach (Autriche), Tagungs publikation, Band 3, pp. 307-319.

Veylon G, Ghestem M, Stokes A, Bernard A. 2015. Quantification of mechanical and hydric components of soil reinforcement by plant roots. Can Geotech J 52: 1839-1849. DOI:10.1139/cgj-2014-0090

Cite this article as: Alain Bernard, Laurent Peyras, Paul Royet, Casagrande large shear box test: an experimental test equipment to evaluate the properties of coarse soils and for other applications in geomechanics, Rev. Fr. Geotech. 2016, 146, 4. 